\title{
Three-dimensional Anosov flag manifolds
}

\author{
THIERRY BARBOT
}

\begin{abstract}
Let $\Gamma$ be a surface group of higher genus. Let $\rho_{0}: \Gamma \rightarrow \operatorname{PGL}(V)$ be a discrete faithful representation with image contained in the natural embedding of $\operatorname{SL}(2, \mathbb{R})$ in $\operatorname{PGL}(3, \mathbb{R})$ as a group preserving a point and a disjoint projective line in the projective plane. We prove that $\rho_{0}$ is $(G, Y)$-Anosov (following the terminology of Labourie [15]), where $Y$ is the frame bundle. More generally, we prove that all the deformations $\rho: \Gamma \rightarrow \operatorname{PGL}(3, \mathbb{R})$ studied in our paper [2] are $(G, Y)$-Anosov. As a corollary, we obtain all the main results of [2] and extend them to any small deformation of $\rho_{0}$, not necessarily preserving a point or a projective line in the projective space: in particular, there is a $\rho(\Gamma)$-invariant solid torus $\Omega$ in the flag variety. The quotient space $\rho(\Gamma) \backslash \Omega$ is a flag manifold, naturally equipped with two 1-dimensional transversely projective foliations arising from the projections of the flag variety on the projective plane and its dual; if $\rho$ is strongly irreducible, these foliations are not minimal. More precisely, if one of these foliations is minimal, then it is topologically conjugate to the strong stable foliation of a double covering of a geodesic flow, and $\rho$ preserves a point or a projective line in the projective plane. All these results hold for any $(G, Y)$-Anosov representation which is not quasi-Fuchsian, ie, does not preserve a strictly convex domain in the projective plane.
\end{abstract}

$57 \mathrm{M} 50$

\section{Introduction}

A flag is a pair $(p, d)$ where $p$ is the point of the projective plane and $d$ a projective line containing $p$. The group $G=\operatorname{PGL}(3, \mathbb{R})$ of projective transformations of the projective plane acts naturally on the flag variety $X$, ie, the space of flags.

Let $\Gamma$ be the fundamental group of a closed surface $\Sigma$ of higher genus. In [2], we considered representations $\rho: \Gamma \rightarrow G$ near horocyclic representations, ie, obtained from a faithful discrete representation $\Gamma \rightarrow H=\operatorname{SL}(2, \mathbb{R})$ composed with the natural morphism identifying $H$ with the commutator subgroup of the stabilizer in $G$ of a point $p_{0}$ and a projective line $d_{0}$ in the projective plane, with $p_{0} \notin d_{0}$. Actually, in [2], we only considered some deformations of horocyclic representations that we called hyperbolic representations. They are characterized by the following properties: 
- They are radial representations, ie $p_{0}$ is still a global fixed point, and the induced projective action on the space $D \approx \mathbb{R} \mathbb{P}^{1}$ of 2 -planes in $\mathbb{R}^{3}$ containing the projective line $p_{0}$ is the action induced by a discrete faithful representation of $\Gamma$ into $\operatorname{SL}(2, \mathbb{R})$.

- Moreover, $p_{0}$ is a fixed point of $\rho(\gamma)$ of saddle type for every nontrivial element $\gamma$ of $\Gamma$.

We proved that for such a representation, there is a closed $\rho(\Gamma)$-invariant simple closed curve $\Lambda$ in $X$, and a open $\rho(\Gamma)$-invariant domain $\Omega$, both depending on $\rho$, such that:

- $\Lambda$ is the image of a one-to-one continuous $\Gamma$-equivariant map from the projective line $\mathbb{R} P^{1}$ into $X$, where the action of $\Gamma$ on $\mathbb{R} P^{1}$ is the usual projective action (which is unique up to topological conjugacy).

- The action of $\rho(\Gamma)$ on $\Omega$ is free and properly discontinuous. The quotient space of this action, called $M$, is a flag manifold (cf Section 2.2). The first tautological foliation (see Section 6.1) is topologically conjugate to the strong stable foliation of a double covering of the geodesic flow on $\Sigma$. On the other hand, the second tautological foliation is not minimal, except when $\rho(\Gamma)$ also preserves a projective line.

In the present paper, we extend all these results omitting the assumption that $\rho(\Gamma)$ admits a global fixed point. The key observation is that horocyclic representations are $(G, Y)$-Anosov in the terminology of Labourie [15], where $Y$ is the frame variety, ie, the space of noncollinear points in the projective plane (we recall the definition of $(G, Y)$-Anosov representations in Section 2.5).

Among $(G, Y)$-Anosov representations, special attention has been be given to hyperconvex representations, ie, those preserving a strictly convex domain of the projective plane. S Choi and W Goldman [7] proved that every representation $\rho: \Gamma \rightarrow G$ which is quasi-Fuchsian, ie, which can be continuously deformed to a representation taking values in $\operatorname{SO}_{0}(1,2) \subset \operatorname{PGL}(3, \mathbb{R})$, preserves such a strictly convex curve. It follows easily that they are $(G, Y)-$ Anosov, hence, hyperconvex. F Labourie [15] has extended this result to the higher-dimensional case. In [12], O Guichard proved that conversely, any hyperconvex representation is quasi-Fuchsian.

In the present paper, our first interest is in nonhyperconvex $(G, Y)$-Anosov representations. The key observation, allowing to recover the results of [2], is:

Theorem 1.1 Hyperbolic representations are $(G, Y)$-Anosov. More precisely, a radial representation is $(G, Y)-$ Anosov if and only if it is hyperbolic. 
Our guess is that nonhyperconvex $(G, Y)$-Anosov representations form a connected space (see Question 2 in Section 8). Observe that they all belong to the same connected component of the space of representations of $\Gamma$ into $G$ : the component of the trivial representation (Corollary 6.7), whereas hyperconvex representations are those in the Hitchin component (see Sections 2.6 and 2.5.2).

$(G, Y)$-Anosov representations, hyperconvex or not, share common properties. In Section 3, we prove that there is a $\rho(\Gamma)$-invariant Jordan curve $L$ in the projective plane, and also a $\rho(\Gamma)$-invariant Jordan curve $L^{*}$ in the dual projective plane (ie the space of projective lines in the projective plane). The combination of $L$ and $L^{*}$ provides an invariant curve $\Lambda$ in $X$, called the limit curve, which can be defined as the set of flags $(p, d)$ where $p$ belongs to $L$ and $d$ to $L^{*}$.

Let $\Omega$ be the open domain in $X$ comprising flag $(p, d)$ where $p$ is not in $L$ and $d$ not in $L^{*}$. In Section 5, we prove:

Theorem 1.2 For any $(G, Y)$-Anosov representation $\rho: \Gamma \rightarrow G$, the action of $\rho(\Gamma)$ on $\Omega$ is free and properly discontinuous.

The quotient $M=\rho(\Gamma) \backslash \Omega$ is a natural flag manifold, which we call an Anosov flag manifold. We can restate part of Theorem 1.2 by saying that $(G, Y)$-Anosov representations are holonomy representations of flag manifolds.

We insist on the low regularity of the invariant curves $L, L^{*}$ : in the nonhyperconvex case, each of them is Hölder continuous, but non-Lipschitz except if it is a projective line. It follows that when $\rho$ is irreducible (ie preserves no point and no line in the projective plane) then $\Lambda$ is not Lipschitz regular (see Corollary 7.2) ${ }^{1}$.

The flag variety admits two $G$-invariant foliations by circles, that we call tautological foliations. The first (respectively the second) is the one admitting as leaves the flags $(p, d)$ where $p$ (respectively $d$ ) is fixed. They induce on every flag manifold, in particular the Anosov flag manifold $M=\rho(\Gamma) \backslash \Omega$, two transversely projective 1dimensional foliations, that we still call first and second tautological foliations (see Section 6.1). When $\rho$ is hyperconvex, $M$ admits three connected components, with well-understood geometrical features (see Remark 3.17), and tautological foliations with well-identified dynamical properties (see Remark 6.2): they are either foliations by circles or doubly covered by the geodesic flow on $\Sigma$ for any hyperbolic metric.

\footnotetext{
${ }^{1}$ A similar statement is true in the hyperconvex case: the limit curve is in general $C^{1}$ with Hölder derivatives, but if the derivatives are Lipschitz, then $\rho(\Gamma)$ preserves a conic, ie, is conjugate in $\operatorname{PGL}(3, \mathbb{R})$ to a Fuchsian subgroup.
} 
The situation when $\rho$ is not hyperconvex is completely different (cf Section 6.4): the tautological foliations in this case are never foliations by circles or finitely covered by Anosov flows. In the case of horocyclic representations, the tautological foliations are the horocyclic foliations associated to the geodesic flow of $\Gamma \backslash \mathbb{H}^{2}$. More generally, if $L$ and $L^{\prime}$ are both projective lines, then the tautological foliations are the strong stable and unstable foliations of some Desarguian Anosov flow (see Section 6.2). In particular, these foliations are minimal and uniquely ergodic.

The situation can be summarized as follows.

Theorem 1.3 Let $\rho$ be a $(G, Y)-$ Anosov representation, which is not hyperconvex.

- If $L$ (respectively $L^{*}$ ) is a projective line, then the second (respectively first) tautological foliation is topologically conjugate to the horocyclic flow of a Desarguian Anosov flow,

- If $L$ is not a projective line, then the second tautological foliation is not minimal.

The dynamical properties of these foliations are quite interesting. We suspect that the tautological foliations never admit periodic orbits (see Question 5 in Section 8). If our suspicion is confirmed, it would provide examples of flows with unusual behavior. For example, recall the Seifert Conjecture, asserting that any flow on the three-dimensional sphere admits a periodic orbit. The first smooth counterexamples to this conjecture were found by K Kuperberg [14]. Observe moreover that the tautological foliations considered here can be volume-preserving: for example, this is the case for second tautological foliations associated to hyperbolic radial representations for which the morphism $u: \Gamma \rightarrow \mathbb{R}$ is trivial (see Section 4.2). As far as we know, the only known examples of volume-preserving flows on the 3-sphere without periodic orbits have regularity at most $C^{2}$ by G Kuperberg [13] and V L Ginzburg and B Z Gürel [10]. As a matter of fact, volume-preserving flows on 3-manifolds which are not minimal and without periodic orbits are quite uncommon; hence, it seems to us quite interesting to answer our Question 5.

Acknowledgements I would like to thank F Labourie, who made me aware of the $(G, Y)$-Anosov character of horocyclic representations. G Kuperberg has corrected some imprecisions in a previous version of this paper regarding counterexamples to the Seifert conjecture. 


\section{Definitions}

\subsection{The flag variety}

Let $V$ be the vector space $\mathbb{R}^{3}$, and $\left(e_{1}, e_{2}, e_{3}\right)$ its canonical basis. Let $V^{*}$ be the dual vector space, with the dual canonical basis $\left(e_{1}^{*}, e_{2}^{*}, e_{3}^{*}\right)$. We denote by $\left\langle v \mid v^{*}\right\rangle$ the evaluation of an element $v^{*}$ of $V^{*}$ on an element $v$ of $V$.

Let $N(v), N\left(v^{*}\right)$ denote the norms on $V, V^{*}$, respectively, for the Euclidean metrics on $V, V^{*}$ in which the canonical basis is orthonormal.

$P(V)$ and $P\left(V^{*}\right)$ are the associated projective spaces. Elements of $P(V)$ are denoted $[v]$.

The flag variety $X$ is the closed subset of $P(V) \times P\left(V^{*}\right)$ formed by pairs $\left([v],\left[v^{*}\right]\right)$ satisfying $\left\langle v \mid v^{*}\right\rangle=0$.

$G$ is the group $\operatorname{SL}(V) \approx \operatorname{PGL}(V)$. The group $G$ acts naturally on $V$ and admits a dual (left) action on $V^{*}$ uniquely defined by requiring $\left\langle u \mid g \cdot v^{*}\right\rangle=\left\langle g^{-1} u \mid v^{*}\right\rangle$ for any $u, v^{*}$. For any $g$ in $G=\operatorname{SL}(V)$, we denote by $g^{*}$ the corresponding element of $\operatorname{SL}\left(V^{*}\right)$. If $\operatorname{SL}(V)$ and $\operatorname{SL}\left(V^{*}\right)$ are identified with $\operatorname{SL}(3, \mathbb{R})$ via the canonical basis, $g^{*}$ is the inverse of the transpose of $g$.

The diagonal action restricts as a natural action of $G$ on $X$.

Remark 2.1 Every element of $P\left(V^{*}\right)$ defines a projective line in $P(V)$ (the projection to $P(V)$ of its kernel). Dually, every element of $P\left(V^{*}\right)$ corresponds to a projective line in $P(V)$. Hence, we can consider $P(V)$ to be the space of projective lines of $P\left(V^{*}\right)$ and $P\left(V^{*}\right)$ to be the space of projective lines of $P(V)$.

The image we have in mind is to view an element of $X$ as a flag, ie, a point in the projective plane and a projective line containing this point.

In order to formalize this point of view, we introduce the following notation: if $K$ is a subspace of $V$ (resp. of $V^{*}$ ), we denote by $K^{\perp}$ its orthogonal, ie, the subset of $V^{*}$ (resp. of $V$ ) vanishing on $K$. Hence, for any $[v]$ in $P(V),\left[v^{\perp}\right]$ is the corresponding projective line in $P\left(V^{*}\right)$.

If $[u],[v]$ are distinct elements of $P(V)$, we denote by $\left[(u v)^{*}\right]$ the element $\left[K^{\perp}\right]$ of $P\left(V^{*}\right)$, where $K$ is the 2-dimensional space spanned by $u$ and $v$. We employ similar notation when $[u],[v]$ belong to $P\left(V^{*}\right)$.

Remark 2.2 Let $P^{+}$(respectively $P^{-}$) be the subgroup of $G$ containing the upper (respectively lower) triangular matrices (under the identification of $G$ with $\operatorname{SL}(3, \mathbb{R})$ ). 
Let $X^{ \pm}$be the quotient spaces $G / P^{ \pm}$: the map $g \mapsto\left(\left[g e_{1}\right],\left[g^{*} e_{3}^{*}\right]\right)$ induces the identification $X^{+}=G / P^{+} \approx X$, and the map $g \mapsto\left(\left[g e_{3}\right]\right.$, [g* $\left.\left.g_{1}^{*}\right]\right)$ induces the identification $X^{-}=G / P^{-} \approx X$. These identifications are $G$-equivariant.

\subsection{Flag manifolds}

A flag structure is a $(G, X)$-structure, for $(G, X)$ as above. We briefly present this notion here. For a more complete description of $(G, X)$-structures, see Thurston [18] or Goldman [11].

A flag structure on a manifold $M$ is an atlas on $M$ with charts taking values in $X$ and coordinates changes expressed in the charts by restrictions of elements of $G$. A typical example of a flag structure is the quotient of an open domain $\Omega$ of $X$ by a discrete subgroup of $G$ acting freely and properly discontinuously on $\Omega$; in particular, the flag variety $X$, itself, is an example. Less trivial examples are given in Sections 2.5.5 and 6. A flag map between flag manifolds is a map which can be locally expressed in the flag charts by restrictions of elements of $G$. A flag map is always a local homeomorphism. A flag map which is bijective is called a flag isomorphism. A flag manifold is an isomorphism class of flag structures on the manifold.

Let $p: \widetilde{M} \rightarrow M$ be the universal covering and $\widetilde{\Gamma}$ the fundamental group of $M$, viewed as the group of covering automorphisms of $p$. For any flag structure on $M$, there is a map $\mathcal{D}: \widetilde{M} \rightarrow X$, called the developing map, and a representation $\rho: \widetilde{\Gamma} \rightarrow G$, called holonomy representation, such that:

- the maps $p$ and $\mathcal{D}$ are flag maps,

- $\mathcal{D}$ is $\widetilde{\Gamma}$-equivariant:

$$
\forall \gamma \in \widetilde{\Gamma} \quad \mathcal{D} \circ \gamma=\rho(\gamma) \circ \mathcal{D}
$$

\subsection{The frame variety}

Let $Y$ denote the frame variety, ie, the space of triples $([u],[v],[w])$ of noncollinear elements of $P(V)$. This 6-dimensional space is homogeneous under the diagonal action of $G$ : it can be identified with the right quotient $G / Z$, where $Z$ is the group of diagonal matrices.

The frame variety admits two natural projections $\pi_{ \pm}$on $X$, defined by (see Remark 2.1 for the notation):

$$
\pi_{+}([u],[v],[w])=\left([u],\left[(u v)^{*}\right]\right) ; \pi_{-}([u],[v],[w])=\left([w],\left[(w v)^{*}\right]\right)
$$

These projections are both $G$-equivariant. Together, they define a map $\Pi: Y \rightarrow X \times X$. We denote by $\mathcal{Y}$ the image of $\Pi$. 
Lemma 2.3 The elements of $\mathcal{Y}$ are the pairs $\left(\left([u],\left[u^{*}\right]\right),\left([v],\left[v^{*}\right]\right)\right)$ satisfying:

- $\left\langle u \mid u^{*}\right\rangle=0$,

- $\left\langle v \mid v^{*}\right\rangle=0$,

- $\left\langle u \mid v^{*}\right\rangle \neq 0$,

- $\left\langle v \mid u^{*}\right\rangle \neq 0$.

In other words, $[u]$ (respectively $[v]$ ) must belong to the projective line $\left[u^{*}\right]\left(\operatorname{resp} .\left[v^{*}\right]\right)$ since we are considering elements of $X$, but it cannot belong to the projective line $\left[v^{*}\right]$ (respectively $\left[u^{*}\right]$ ).

The fibers of the projections $\pi_{ \pm}$are the leaves of $G$-invariant foliations $\mathcal{G}^{ \pm}$of $X$. Let $E^{ \pm}$be the tangent bundles to these foliations. We obtain a $G$-invariant decomposition $T Y=E^{+} \oplus E^{-}$of the tangent bundle of $Y$.

Remark 2.4 The inclusions $Z \subset P^{ \pm}$defines canonical maps $G / Z \rightarrow X^{ \pm}$, which, via the identifications $X^{ \pm} \approx X$ presented in Remark 2.2 and $G / Z \approx Y$, are the maps $\pi_{ \pm}$.

\subsection{The geodesic flow as an Anosov flow}

Let $\Sigma$ be a closed surface with negative Euler characteristic, and $\Gamma$ the fundamental group of $\Sigma$. Select a one-to-one morphism 1: $\Gamma \rightarrow H$ with discrete image, where $H$ denotes the group $\operatorname{SL}(2, \mathbb{R})$. This induces a Fuchsian representation $\overline{1}: \Gamma \rightarrow \bar{H}$, where $\bar{H}$ denotes the group $\operatorname{PSL}(2, \mathbb{R})$ (observe that conversely, any Fuchsian representation lifts to a representation into $H$, since the associated Euler class is even).

Consider the flow on $H$ induced by the right action of the 1-parameter group $A=\left\{a^{t}\right\}$, where:

$$
a^{t}=\left(\begin{array}{cc}
e^{t} & 0 \\
0 & e^{-t}
\end{array}\right)
$$

This flow induces another flow on $M=1(\Gamma) \backslash H$, denoted by $\Phi^{t}$. Its projection on $\bar{M}=\overline{1}(\Gamma) \backslash \bar{H} \approx T^{1} \Sigma$ is a flow $\bar{\Phi}^{t}$.

Remark 2.5 This flow divided by 2 , ie, the flow $p \mapsto \Phi^{t / 2}(p)$, is the familiar geodesic flow associated to the Riemannian surface $\overline{1}(\Gamma) \backslash \mathbb{H}^{2} \approx \Sigma$, where $\mathbb{H}^{2}$ is the hyperbolic plane. 
Consider the following 1-parameter subgroups of $H$ :

$$
h_{+}^{s}=\left(\begin{array}{ll}
1 & s \\
0 & 1
\end{array}\right), \quad h_{-}^{s}=\left(\begin{array}{ll}
1 & 0 \\
s & 1
\end{array}\right)
$$

We have the identities:

$$
h_{+}^{s} a^{t}=a^{t} h_{+}^{\exp (-2 t) s}, \quad h_{-}^{s} a^{t}=a^{t} h_{-}^{\exp (2 t) s}
$$

Denote also by $h_{ \pm}^{s}$ the flow on $M$ induced by the right actions of these 1-parameter subgroups. The flow $h_{+}^{s}$ (resp. $h_{-}^{s}$ ) is called the stable horocyclic flow (resp. unstable horocyclic flow). The flow $\Phi^{t}$ permutes the orbits of $h_{ \pm}^{s}$. Moreover, the orbits of $h_{+}^{s}$ are exponentially contracted by $\Phi^{t}$, whereas the orbits of $h_{-}^{s}$ are exponentially expanded by $\Phi^{t}$. This feature establishes precisely that $\Phi^{t}$ and $\bar{\Phi}^{t}$ are Anosov.

Definition 2.6 An Anosov flow on a closed manifold $M$ equipped with a Riemannian metric $\|$ is a nonsingular flow $\Phi^{t}$ such that the differential of $\Phi^{t}$ preserves a decomposition $T M=\Delta \oplus E^{s s} \oplus E^{u u}$ of the tangent bundle, satisfying the following properties, for some positive constants $a, b$ :

- The line bundle $\Delta$ is tangent to the flow.

- For any vector $v$ in $E^{s s}$ over a point $x$ of $M$, and for any positive $t$ :

$$
\left\|D_{x} \Phi^{t}(v)\right\| \leq b e^{-a t}\|v\|
$$

- For any vector $v$ in $E^{u u}$ over a point $x$ of $M$, and for any negative $t$ :

$$
\left\|D_{x} \Phi^{t}(v)\right\| \leq b e^{a t}\|v\|
$$

2.4.1 Stable and unstable leaf spaces Denote by $\mathcal{A}_{ \pm}$the group generated by $a^{t}$ and $h_{ \pm}^{s}$. It is isomorphic to the group of volume-preserving affine transformations of the plane. The orbits on $M$ or $\bar{M}$ of $\mathcal{A}_{+}$are called stable leaves; the orbits of $\mathcal{A}_{-}$are called unstable leaves. We denote by $\mathcal{S}_{ \pm}, \overline{\mathcal{S}}_{ \pm}$the quotient spaces $H / A_{ \pm}$, $\bar{H} / \mathcal{A}_{ \pm}$. The latter, $\overline{\mathcal{S}}_{ \pm}$, are both homeomorphic to the projective line $\mathbb{R} P^{1}$, and $\mathcal{S}_{ \pm}$ are double coverings of $\overline{\mathcal{S}}_{ \pm}$. Moreover, these identifications are $H$-equivariant, where the $H$-action on $\overline{\mathcal{S}}_{ \pm}$is the action induced by left translation, and the $H$-action on $\mathbb{R} P^{1}$ is the usual projective action.

2.4.2 The bifoliated orbit space The map $h A \mapsto\left(h \mathcal{A}_{+}, h \mathcal{A}_{-}\right)$embeds the orbit space $\bar{Q}=\bar{H} / a^{t}$ into the torus $\overline{\mathcal{S}}_{+} \times \overline{\mathcal{S}}_{-}$. More precisely, for fixed $H$-equivariant identifications $\overline{\mathcal{S}}_{ \pm} \approx \mathbb{R} P^{1}$, the image of this embedding is the complement in $\mathbb{R} P^{1} \times$ $\mathbb{R} P^{1}$ of the diagonal $\Delta$. In other words, every $\mathcal{A}_{+}$-orbit $x$ intersects every $\mathcal{A}_{-}$-orbit, 
except one, which we call $\alpha(x)$. Note that we have defined a continuous $\Gamma$-equivariant $\operatorname{map} \alpha: \overline{\mathcal{S}}_{+} \rightarrow \overline{\mathcal{S}}_{-}$.

We denote by $\overline{\mathcal{Q}}$ the image of $\bar{Q}$ in $\overline{\mathcal{S}}_{+} \times \overline{\mathcal{S}}_{-}$; this is the complement of the graph of $\alpha: \overline{\mathcal{S}}_{+} \rightarrow \overline{\mathcal{S}}_{-}$.

\subsection{Anosov representations}

Let $\rho: \Gamma \rightarrow G$ be any representation, and let $\pi_{\rho}: E_{\rho} \rightarrow M$ be the associated flat $(G, Y)$-bundle: $E_{\rho}$ is the quotient of $H \times Y$ by the relation identifying each $(h, y)$ with $(1(\gamma) h, \rho(\gamma) . y)$, for every $\gamma$ in $\Gamma$. The projection $(h, y) \mapsto h$ induces a map $\pi_{\rho}$ from $E_{\rho}$ onto $M=1(\Gamma) \backslash H$, which is a $G$-bundle, with fiber $Y$.

The (trivial) foliation of $H \times Y$ having as leaves the fibers of $(h, y) \mapsto y$ induces a foliation on $M$, which we denote by $\mathcal{F}_{\rho}$, and call the horizontal foliation. The leaves of $\mathcal{F}_{\rho}$ are transverse to the fibers of $\pi_{\rho}$.

The flow $\Phi^{t}$ lifts uniquely to a horizontal flow $\Phi_{\rho}^{t}$ in $E_{\rho}$, ie, tangent to the horizontal foliation: just take the flow induced in the quotient by the flow on $H \times Y$ defined by $(h, y) \mapsto\left(h a^{t}, y\right)$.

We have defined foliations $\mathcal{G}^{ \pm}$on $Y$. They provide two 3-dimensional foliations on $H \times Y$ and induce on $E_{\rho}$ two 3-dimensional foliations $\mathcal{F}^{ \pm}$which are preserved by $\Phi_{\rho}^{t}$ and tangent to the fibers of $\pi_{\rho}$.

We will mainly consider the tangent bundles $E_{\rho}^{ \pm}$of these foliations, which are canonically induced by the bundles $E^{ \pm}$over $Y$.

Definition $2.7[15]$ A $(G, Y)$-Anosov structure over $\left(M, \Phi^{t}\right)$ is the data of a representation $\rho: \Gamma \rightarrow G$ and a continuous section $s$ of $\pi_{\rho}$ with the following properties:

- The image $S$ of $s$ is $\Phi_{\rho}^{t}$-invariant.

- For any norm $\|$ on $E_{\rho}$, there are positive constants $a$ and $b$ such that for any $p$ in $S$, any positive $t$, any vector $v^{+}$in $E_{\rho}^{+}$over $p$, and any vector $v^{-}$in $E_{\rho}^{-}$ over $p$, we have:

$$
\begin{aligned}
\left\|D_{p} \Phi_{\rho}^{t}\left(v^{+}\right)\right\| & \leq b e^{-a t}\left\|v^{+}\right\| \\
\left\|D_{p} \Phi_{\rho}^{-t}\left(v^{-}\right)\right\| & \leq b e^{-a t}\left\|v^{-}\right\|
\end{aligned}
$$

If these conditions are fulfilled, then the representation $\rho$ is said to be $(G, Y)$-Anosov, or an Anosov flag representation. 
Remark 2.8 Here, $Y$ is the frame variety, and $G$ the group $\operatorname{SL}(V)$, but, of course, Definition 2.7 extends to any other pair $(G, Y)$, and any Anosov flow can play the role played here by the "geodesic flow" $\Phi^{t}$.

The section $s$ appearing in this definition is not assumed to be differentiable, even nor Lipschitz. The maximal regularity which can be required in general is Hölder continuity.

The main interest of Anosov representations is their stability: it follows from the structural stability of Anosov flows (more precisely, of hyperbolic closed sets) that the set of $(G, Y)$-Anosov representations is an open domain in the space of representations of $\Gamma$ into $G$ equipped with its natural topology (see Proposition 2.1 of [15]). Observe that the perturbed $(G, Y)$-Anosov structure covers the same Anosov flow $\left(M, \Phi^{t}\right)$.

Another important feature of Anosov representations is that they provide nice $\Gamma-$ invariant geometric objects, obtained as follows:

The section $s$ of a $(G, Y)$-Anosov structure lifts to a continuous map $f: H \rightarrow Y$ such that:

- $f$ is $\Gamma$-equivariant: $f \circ 1(\gamma)=\rho(\gamma) \circ f$,

- $f$ is invariant by the lifted flow: $f\left(h a^{t}\right)=f(h)$.

Therefore, $f$ induces a continuous $\Gamma$-equivariant map from the orbit space $Q=H / A$ into $Y$.

Lemma 2.9 The restriction of $\pi_{+} \circ f$ (resp. $\pi_{-} \circ f$ ) to any $\mathcal{A}_{+}$-orbit (resp. $\mathcal{A}_{-}$-orbit) is constant.

Proof Let $p, p^{\prime}$ be two elements of $M$ belonging to the same stable leaf, ie, on the same (right) $\mathcal{A}_{+}$-orbit: for positive times $t$, the iterates $\Phi^{t}(p)$ and $\Phi^{t}\left(p^{\prime}\right)$ remain at a bounded distance apart inside the stable leaf. Then the exponential dilatation along the leaves of $\mathcal{F}^{-}$implies that $s(p)$ and $s\left(p^{\prime}\right)$ must belong to the same leaf of $\mathcal{F}^{+}$. The lemma follows.

It follows from Lemma 2.9 that $\pi_{+} \circ f$ and $\pi_{-} \circ f$ induce maps $f_{+}: \mathcal{S}_{+} \rightarrow X$ and $f_{-}: \mathcal{S}_{-} \rightarrow X$. Of course, $f_{ \pm}$are both $\Gamma$-equivariant: their images (which, as we will see, are the same) are $\Gamma$-invariant (a priori only immersed) topological circles in the flag variety. 
2.5.1 Splitting the definition Consider the flat $X$-bundle $\pi_{\rho}^{X}: E_{\rho}(X) \rightarrow M$ associated to $\rho$. The total space $E_{\rho}(X)$ is the quotient of $H \times X$ by the $\Gamma$-action defined by $(h, x) \rightarrow(\gamma h, \rho(\gamma) x)$. The map $\Pi$ defines a fibered embedding $\Pi_{\rho}: E_{\rho} \rightarrow$ $E_{\rho}(X) \times E_{\rho}(X)$. Let $\mathcal{Y}_{\rho}$ be the image of $\Pi_{\rho}$. The flow $\Phi^{t}$ also lifts in a unique way to a horizontal flow $\Phi_{X}^{t}$ on $E_{\rho}(X): \Phi_{X}^{t}(h, x)=\left(h a^{t}, x\right)$. Obviously, Definition 2.7 is equivalent to:

Definition 2.10 A $(G, Y)$-Anosov structure over $\left(M, \Phi^{t}\right)$ is the data of a representation $\rho: \Gamma \rightarrow G$ and two continuous section $s^{ \pm}$of $\pi_{\rho}^{X}$ satisfying the following properties:

- The sections $s^{ \pm}$are preserved by the flows: $\Phi_{X}^{t}\left(s^{ \pm}(p)\right)=s^{ \pm}\left(\Phi^{t} p\right)$.

- The image of $s^{+}$(resp. $s^{-}$) is a (exponentially) repellor (resp. attractor) for $\Phi_{X}^{t}$.

- For every $p$ in $M$, the pair $\left(s_{+}(p), s_{-}(p)\right)$ belongs to $\mathcal{Y}_{\rho}$.

2.5.2 Quasi-Fuchsian representations A very nice family of $(G, Y)$-Anosov representations is the family of quasi-Fuchsian representations (in the terminology of [15]), ie, the representations $\rho: \Gamma \rightarrow G \approx \operatorname{SL}(3, \mathbb{R})$ which are in the Hitchin component, ie, which can be deformed to Fuchsian representations, in other words, to a representation $\rho_{0}: \Gamma \rightarrow \mathrm{SO}_{0}(1,2)$. Indeed, $\mathrm{F}$ Labourie has proven that quasi-Fuchsian representations are $(G, Y)-$ Anosov.

Let's be a bit more precise: S Choi and W Goldman proved in [7] that any representation in the Hitchin component induces an action on $P(V)$ preserving a strictly convex domain $C$. The set of flags $\left([u],\left[v^{*}\right]\right)$ where $[u]$ is a point in $\partial C$ and $\left[v^{*}\right]$ a support projective line of $C$ is a $\rho(\Gamma)$-invariant curve in $X$; in fact, this topological circle is equal to the images $f_{+}\left(\mathcal{S}_{+}\right)$and $f_{-}\left(\mathcal{S}_{-}\right)$. In order to provide a simple hint, we just claim that the fact that this kind of representation is flag Anosov can be inferred from the fact that the geodesic flow associated to the Finsler Hilbert metric on $\rho(\Gamma) \backslash C$ is Anosov.

Following F Labourie, this kind of curve is said to be hyperconvex, and a $(G, Y)-$ Anosov representation preserving a hyperconvex curve is said hyperconvex. Thus, quasi-Fuchsian representations and hyperconvex representations coincide. O Guichard extended recently this statement to all dimensions [12].

2.5.3 Horocyclic representations Consider now another family of $(G, Y)$-Anosov representations, which are not quasi-Fuchsian. They are obtained from an embedding $\rho_{0}$ of $H=\mathrm{SL}(2, \mathbb{R})$ into a subgroup of $G=\operatorname{SL}(V)$ that admits a global fixed point in $P(V) \times P\left(V^{*}\right) \backslash X$. 
For the fixed point, we select here the pair $\left(e_{2}, e_{2}^{*}\right)$. The embedding $\rho_{0}$ is the representation sending the matrix

$$
\left(\begin{array}{ll}
a & b \\
c & d
\end{array}\right)
$$

to the element

$$
\left(\begin{array}{lll}
a & 0 & b \\
0 & 1 & 0 \\
c & 0 & d
\end{array}\right)
$$

of $G \approx \operatorname{SL}(3, \mathbb{R})$ (in the identification arising from the canonical basis).

Recall the notation introduced in Sections 2.1 and 2.3. Observe that $\rho_{0}(A)$ is contained in $Z$, and that every $\rho_{0}\left(h_{ \pm}^{s}\right)$ belongs to $P^{ \pm}$. The adjoint action of $\rho_{0}\left(a^{t}\right)$ on the Lie algebra $\mathfrak{s l}(V)$ of $G$ is diagonalizable, and our choice of $\rho_{0}$ ensures that the subspace spanned by eigenvectors with positive eigenvalues (resp. negative) for $\operatorname{ad}\left(a^{t}\right)$ is the Lie algebra $\mathcal{P}^{+}$(resp. $\mathcal{P}^{-}$) of $P^{+}$(resp. $P^{-}$). Their intersection is the Lie algebra of $Z$.

Theorem 2.11 [15, Proposition 3.1] Let $\bar{\Gamma}$ be any cocompact subgroup of $H$. The restriction of $\rho_{0}$ to $\bar{\Gamma}$ is $(G, Y)-$ Anosov.

Proof See the Appendix.

Remark 2.12 Of course, we will apply Theorem 2.11 to $\bar{\Gamma}=1(\Gamma)$. Actually, from the beginning, we could have selected as $\Gamma$ any discrete cocompact subgroup of $H$, possibly with torsion, but this level of generality requires a little more caution in the formulation of statements, which we considered unnecessary and slightly uncomfortable. The reader should have no difficulty to extend the results of this paper to this more general context.

Definition 2.13 The $(G, Y)$-Anosov representation $\rho_{0} \circ 1$ is a horocyclic representation.

Remark 2.14 Henceforth, except in Proposition 6.3, we will drop the symbol 1, considering $\Gamma$ directly as a discrete subgroup of $H$.

2.5.4 Invariant curves for horocyclic representations Consider a horocyclic representation $\rho_{0}: \Gamma \rightarrow G$. According to Lemma 2.9, there are two $\Gamma$-equivariant maps $f_{ \pm}: \mathcal{S}_{ \pm} \rightarrow X$, coming from a $\Gamma$-equivariant map $f: H \rightarrow Y$. Here, the map $f$ is defined by: $f(h)=\left(\left[\rho_{0}(h) e_{1}\right],\left[e_{2}\right],\left[\rho_{0}(h) e_{3}\right]\right)$ (see the Appendix). Hence, we have: $f_{+}\left(h \mathcal{A}_{+}\right)=\left(\left[\rho_{0}(h) e_{1}\right],\left[\rho_{0}(h)^{*} e_{3}^{*}\right]\right)$, and $f_{-}\left(h \mathcal{A}_{-}\right)=\left(\left[\rho_{0}(h) e_{3}\right],\left[\rho_{0}(h)^{*} e_{1}^{*}\right]\right)$. These two maps have the same image $\Lambda_{0}$, which is the set of pairs $\left([u],\left[u^{*}\right]\right)$ where $[u]$ belongs to the projective line $\left[\left(e_{2}^{*}\right)^{\perp}\right]$ and $\left[u^{*}\right]$ is a projective line containing $\left[e_{2}\right]$ (in a more symmetric formulation, $\left[u^{*}\right]$ belongs to the projective line $\left[e_{2}^{\perp}\right]$ in $P\left(V^{*}\right)$ ). 
We denote by $L_{0}$ the projective line $\left[\left(e_{2}^{*}\right)^{\perp}\right]$ of $P(V)$, and by $L_{0}^{*}$ the projective line $\left[e_{2}^{\perp}\right]$ of $P\left(V^{*}\right)$. We can then reformulate the statement above: a point $\left([u],\left[u^{*}\right]\right)$ in $X$ belongs to the curve $\Lambda_{0}$ if and only if $[u]$ belongs to $L_{0}$ and $\left[u^{*}\right]$ belongs to $L_{0}^{*}$. Observe that for every $[u]$ in $L_{0}$ there is one and only one element $\left[u^{*}\right]$ of $L_{0}^{*}$ containing $[u]$ : the projective line containing $[u]$ and $\left[e_{2}\right]$.

Observe also that $\Lambda_{0}$ is the closure of the set of attractive fixed points of elements of $\rho_{0}(\Gamma)$ in $X$.

Finally, the image of $f$ is the space of triples $\left([u],\left[e_{2}\right],[w]\right)$ of elements of $P(V)$ where $[u]$ and $[w]$ are distinct elements of $L_{0}$.

2.5.5 Canonical Anosov flag manifolds. Consider the orbits of $\rho_{0}(H)$ in $X$ : there is one 1-dimensional orbit, the curve $\Lambda_{0}$. There are two 2-dimensional orbits:

- the orbit $A_{0}$ containing the points $\left([u],\left[u^{*}\right]\right)$ where $[u]$ belongs to $L_{0}$ and $\left[u^{*}\right]$ does not belong to $L_{0}^{*}$,

- the orbit $A_{0}^{*}$ containing the points $\left([u],\left[u^{*}\right]\right)$ where $[u]$ does not belong to $L_{0}$ and $\left[u^{*}\right]$ belongs to $L_{0}^{*}$.

There is one open orbit: $\Omega_{0}=\left\{\left([u],\left[u^{*}\right]\right) \in X /[u] \notin L_{0},\left[u^{*}\right] \notin L_{0}^{*}\right\}$.

The closures $T_{0}, T_{0}^{*}$ of $A_{0}, A_{0}^{*}$ are Klein bottles, the intersection $T_{0} \cap T_{0}^{*}$ is $\Lambda_{0}$, and $\Omega_{0}$ is the complement in $X$ of $T_{0} \cup T_{0}^{*}$.

The action of $\rho_{0}(H)$ on $\Omega_{0}$ is simply transitive. It provides an identification $\Omega_{0} \approx H$. Therefore, the manifold $M=\Gamma \backslash H$ is homeomorphic to the quotient of $\Omega_{0}$ by $\rho_{0}(\Gamma)$. It provides a natural flag structure on $M$.

Definition 2.15 The quotient $\rho_{0}(\Gamma) \backslash \Omega_{0}$ is a canonical Anosov flag manifold.

\subsection{Existence of deformations}

Consider the space $\operatorname{Rep}(\Gamma, G)$ of representations of the surface group into $G$, modulo inner automorphisms of $G$ on the target.

Theorem 2.16 (Hitchin) $\operatorname{Rep}(\Gamma, G)$ has three connected components.

Let's briefly discuss each of these 3 components:

- (The Hitchin component) This is the component containing the Fuchsian representations. Elements of this component are represented by quasi-Fuchsian representations (see Section 2.5.2). 
- (The trivial component) This is the component containing the trivial representation.

- Representations in the third component are characterized by the fact that they do not lift to representations from $\Gamma$ into the double covering $P^{+} \mathrm{GL}(V)$ of $G$.

Horocyclic representations are not quasi-Fuchsian, and they clearly lift to representations in $\operatorname{GL}(V)$ : hence, they belong to the trivial component. It follows immediately that they can be deformed to representations in $G$ which are not horocyclic! But there are much more elementary ways to prove this statement: any horocyclic representation $\rho_{0}$ can be deformed to a strongly irreducible representation, ie, with image containing no finite index subgroup stabilizing a point or a projective line in $P(V)$ (see eg Proposition 3.11 of [2]).

\section{General properties of $(G, Y)$-Anosov representations}

Let $\rho: \Gamma \rightarrow G$ be any $(G, Y)$-Anosov representation. According to Lemma 2.9, there is a $G$-equivariant map $f: H \rightarrow Y$, inducing $G$-equivariant maps $f_{ \pm}: \mathcal{S}_{ \pm} \rightarrow X$.

Lemma 3.1 For any $\gamma$ in $\Gamma$ and any attractive fixed point $x$ of $\gamma$ in $\mathcal{S}_{ \pm}$, the image of $x$ by $f_{ \pm}$is an attractive (resp, repulsive) fixed point of $\rho(\gamma)$ in $X$.

Proof Quite straightforward. See Proposition 3.2 of [15].

Proposition 3.2 The representation $\rho$ is discrete and faithful. For every nontrivial $\gamma$ in $\Gamma$, the image $\rho(\gamma)$ is loxodromic, ie, admits three eigenvalues with distinct norms.

Proof Except for the discreteness, the proposition follows immediately from Lemma 3.1 , the fact that any nontrivial element of $\Gamma$ admits an attractive fixed in $\mathcal{S}_{+}$, and the fact that loxodromic elements of $\operatorname{SL}(V)$ are precisely elements admitting an attractive fixed point in $X$. The discreteness follows by classical arguments. See Labourie [15] for more details.

Actually, loxodromic elements of $G$ have one and only one attractive fixed point in $X$. Since attractive fixed points of elements of $\gamma$ are dense in $\mathcal{S}_{ \pm}$, we obtain:

Corollary 3.3 The maps $f_{ \pm}$have the same image, which is the closure of the set of attractive fixed points of elements of $\rho(\Gamma)$ in $X$.

Definition 3.4 The common image $f_{+}\left(\mathcal{S}_{+}\right)=f_{-}\left(\mathcal{S}_{-}\right)$is denoted by $\Lambda$, and called the limit curve. 
Recall that $\Phi^{t}$ is a double covering of the geodesic flow: there is a double covering between the associated leaf spaces $\mathcal{S}_{ \pm} \rightarrow \overline{\mathcal{S}}_{ \pm}$. Let $\bar{\tau}$ be the Galois automorphism of this double covering. For any nontrivial element $\gamma$ of $\Gamma$ and any attractive fixed point $x$ of $\gamma$ in $\mathcal{S}_{ \pm}$, the image $\bar{\tau}(x)$ is an attractive fixed point of $\gamma$. By uniqueness of attractive fixed points in $X$, and according to Lemma 3.1, we have $f_{ \pm}(x)=f_{ \pm}(\bar{\tau}(x))$. By density of attractive fixed points in $\mathcal{S}_{ \pm}$, we obtain $f_{ \pm}=f_{ \pm} \circ \bar{\tau}$. Hence:

Corollary 3.5 The maps $f_{ \pm}$induce maps $\bar{f}_{ \pm}: \overline{\mathcal{S}}_{ \pm} \rightarrow X$.

According to Section 2.4.2, the orbit space $\bar{Q}$ can be identified with the complement $\overline{\mathcal{Q}}$ in $\overline{\mathcal{S}}_{+} \times \overline{\mathcal{S}}_{-}$of the graph of a homeomorphism $\alpha: \overline{\mathcal{S}}_{+} \rightarrow \overline{\mathcal{S}}_{-}$. The maps $\bar{f}_{ \pm}$induce a map $\bar{F}: \overline{\mathcal{Q}} \rightarrow X \times X$.

Lemma 3.6 The image of $\bar{F}$ is contained in the image $\mathcal{Y}$ of $\Pi$.

Proof Indeed, the maps $\bar{f}_{ \pm}$arise from a map $f: H \rightarrow Y$.

Lemma 3.7 We have the identity $\bar{f}_{+}=\bar{f}_{-} \circ \alpha$.

Proof Let $x$ in $\overline{\mathcal{S}}_{+}$and $y$ in $\overline{\mathcal{S}}_{-} \backslash\{\alpha(x)\}$. Then $(x, y)$ belongs to the open set $\overline{\mathcal{Q}} \subset \overline{\mathcal{S}}_{+} \times \overline{\mathcal{S}}_{-}$. The image of $(x, y)$ by $\bar{F}$ belongs to $\mathcal{Y}$ (Lemma 3.6). According to Lemma 2.3 , we have $\bar{f}_{-}(y) \neq \bar{f}_{+}(x)$. Hence, $\bar{f}_{+}(x)$ belongs to $\Lambda \backslash \bar{f}_{-}\left(\overline{\mathcal{S}}_{-} \backslash\{\alpha(x)\}\right)$. But $\Lambda \backslash \bar{f}_{-}\left(\overline{\mathcal{S}}_{-} \backslash\{\alpha(x)\}\right)$ is either empty or reduced to $\left\{\bar{f}_{-}(\alpha(x))\right\}$. The lemma follows.

In the proof above, we have shown in particular that $\Lambda \backslash \bar{f}_{-}\left(\overline{\mathcal{S}}_{-} \backslash\{\alpha(x)\}\right)$ is not empty. Hence:

Corollary 3.8 The maps $\bar{f}_{+}$and $\bar{f}_{-}$are injective.

The flag manifold $X$ is a closed subset of $P(V) \times P\left(V^{*}\right)$. Let $\eta_{ \pm}\left(\right.$resp. $\eta_{ \pm}^{*}$ ) be the composition of $\bar{f}_{ \pm}$with the projection of $X$ on $P(V)$ (resp. $P\left(V^{*}\right)$ ).

Lemma 3.9 The maps $\eta_{ \pm}: \overline{\mathcal{S}}_{ \pm} \rightarrow P(V)$ and the maps $\eta_{ \pm}^{*}: \overline{\mathcal{S}}_{ \pm} \rightarrow P\left(V^{*}\right)$ are injective.

Proof We only deal with $\eta_{+}$; the other cases are similar. Let $x, x^{\prime}$ be two elements of $\overline{\mathcal{S}}_{+}$with the same image by $\eta_{+}$: if $[u]=\eta_{+}(x)$, then $\bar{f}_{+}(x)=\left([u],\left[u^{*}\right]\right)$, and $\bar{f}_{+}\left(x^{\prime}\right)=\left([u],\left[v^{*}\right]\right)$ for some $\left[u^{*}\right],\left[v^{*}\right]$ in $P\left(V^{*}\right)$ such that $\left\langle u \mid u^{*}\right\rangle=\left\langle u \mid v^{*}\right\rangle=0$. 
Assume $x \neq x^{\prime}$. The pair $(x, y)$, with $y=\alpha\left(x^{\prime}\right)$, is an element of $\overline{\mathcal{Q}} \approx \bar{Q}$. Its image by $\bar{F}$ is $\left(\bar{f}_{+}(x), \bar{f}_{-}\left(\alpha\left(x^{\prime}\right)\right)\right)$, which, according to Lemma 3.7 , is equal to $\left(\bar{f}_{+}(x), \bar{f}_{+}\left(x^{\prime}\right)\right)$. On one hand, this pair must belong to the image $\mathcal{Y}$ of $\Pi$. On the other hand, it has the form $\left(\left([u],\left[u^{*}\right]\right),\left([u],\left[v^{*}\right]\right)\right)$. From the description of the image of $\mathcal{Y} \subset X \times X$ (Lemma 2.3), we obtain a contradiction.

Hence, $x=x^{\prime}$. The lemma is proved.

Definition 3.10 Let $L$ be the image of $\eta_{+}$: this is the image of $\eta_{-}$too. Let $L^{*}$ be the common image of $\eta_{+}^{*}$ and $\eta_{-}^{*}$.

According to Lemma 3.9, $L$ and $L^{*}$ are closed simple curves.

Lemma 3.11 The limit curve $\Lambda$ is the set of pairs $\left([v],\left[v^{*}\right]\right)$ where $[v]$ belongs to $L$ and $\left[v^{*}\right]$ belongs to $L^{*}$.

Proof One of the inclusion is obvious. Conversely, let $\left([v],\left[v^{*}\right]\right)$ be an element of $X$ with $[v] \in L,\left[v^{*}\right] \in L^{*}$. Let $(x, y)$ be the element of $\overline{\mathcal{S}}_{+} \times \overline{\mathcal{S}}_{-}$satisfying:

$$
\eta_{+}(x)=[v], \quad \eta_{-}^{*}(y)=\left[v^{*}\right]
$$

Since $\left\langle v \mid v^{*}\right\rangle=0$, the pair $\left(\bar{f}_{+}(x), \bar{f}_{-}(y)\right)$ does not belong to $\mathcal{Y}$. Therefore, $(x, y)$ cannot belong to $\overline{\mathcal{Q}}$. We have $y=\alpha(x)$.

According to Lemma 3.7, we have $\bar{f}_{+}(x)=\bar{f}_{-}(y)$. Hence, the $P\left(V^{*}\right)$-component of $\bar{f}_{+}(x)$ is $\eta_{-}^{*}(y)=\left[v^{*}\right]$. By construction, the $P(V)$-component of $\bar{f}_{+}(x)$ is $[v]$. Therefore, the pair $\left([v],\left[v^{*}\right]\right)=\bar{f}_{+}(x)$ belongs to $\Lambda$. The lemma follows.

By Lemma 3.11 and Lemma 3.9:

Corollary 3.12 Every projective line in $P(V)$ belonging to $L^{*}$ (ie, of the form $\left[\left(u^{*}\right)^{\perp}\right]$ with $\left[u^{*}\right]$ in $\left.L^{*}\right)$ intersects $L$ in one and only one point.

The dual statement, with $L$ and $L^{*}$ exchanged, is of course true. Hence, the corollary above can be complemented by:

Corollary 3.13 For every point $[u]$ in $L$, there is one and only one projective line of $P(V)$ belonging to $L^{*}$ and containing $[u]$.

Corollary 3.14 The curve $L$ (respectively $L^{*}$ ) is the closure of the set of attractive fixed points in $P(V)$ (respectively $P\left(V^{*}\right)$ ) of elements of $\rho(\Gamma)$ (respectively $\left.\rho^{*}(\Gamma)\right)$ 
Of course, when $\rho$ is a horocyclic representation, the curves $L, L^{*}$ are the projective lines $L_{0}, L_{0}^{*}$. In this case, we defined Klein bottles $T_{0}, T_{0}^{*}$ and an open domain $\Omega_{0}$ (see Section 2.5.5). These constructions extend to the general case in the following way:

Definition 3.15 For any $(G, Y)$-Anosov representations, let $T$ (respectively $\left.T^{*}\right)$ to be the set of flags $\left([u],\left[u^{*}\right]\right.$ ) with $[u] \in L$ (respectively $\left[u^{*}\right] \in L^{*}$ ), and let $\Omega$ be the complement in $X$ of the union $T \cup T^{*}$.

According to Lemma 3.11, the limit curve $\Lambda$ is the intersection $T \cap T^{*}$.

Definition 3.16 The complements of $\Lambda$ in $T, T^{*}$ are denoted $A, A^{*}$.

Remark 3.17 All the results of these sections apply to any $(G, Y)$-Anosov representation and, in particular, to quasi-Fuchsian representations. In this case, $L$ is the boundary of a $\rho(\Gamma)$-invariant convex domain $C$ in $P(V)$, and $L^{*}$ is the boundary of the dual convex $C^{*}$ : elements in $L^{*}$ are projective lines in $P(V)$ tangent to $L$. The sets $T, T^{*}$ are (topological) tori, and $A, A^{*}$ are annuli.

The domain $\Omega$ in this case has 3 connected components:

- One component is the set of flags $\left([v],\left[v^{*}\right]\right)$ with $[v] \in C$ : This component is canonically identified with the projectivized tangent bundle of $C$.

- Another component is the set of flags $\left([v],\left[v^{*}\right]\right)$ with $\left[v^{*}\right] \in C^{*}$ : It is canonically identified with the projectivized tangent bundle of $C^{*}$.

- Finally, there is a third component, consisting of the flags $\left([v],\left[v^{*}\right]\right)$ with $[v] \notin C$, $\left[v^{*}\right] \notin C^{*}$.

The last component, in some way, has a Lorentzian flavor. Indeed, when $\rho$ is Fuchsian, ie, when $C$ and $C^{*}$ are ellipses, this last component is canonically identified with the projectivized bundles of timelike vectors of de Sitter space.

\section{Special deformations}

In this section, we fix the embedding $\Gamma \subset H$, ie, the horocyclic morphism $\rho_{0}: \Gamma \rightarrow G$. The projection of $\Gamma$ in $\bar{H}$ is injective; we still denote by $\Gamma$ the image of this projection. The quotient $\Gamma \backslash \bar{H}$ is the unit tangent bundle of the surface $\Sigma$.

Definition 4.1 For any $\gamma$ in $\Gamma$ let $r(\gamma)$ be the spectral radius of $\gamma \in H$.

Up to the sign, the eigenvalues of $\gamma \in H$ are $r(\gamma), r(\gamma)^{-1}$; when $\gamma$ is nontrivial, we have $r(\gamma)>1$. 


\section{1 $\Lambda$-Preserving deformations}

4.1.1 A flag version of the geodesic flow Consider ${ }^{2}$ the following 1-parameter subgroup of $G$ :

$$
\varphi^{t}=\left(\begin{array}{ccc}
e^{t / 3} & 0 & 0 \\
0 & e^{-2 t / 3} & 0 \\
0 & 0 & e^{t / 3}
\end{array}\right)
$$

It commutes with every $\rho_{0}(h)$. Hence, it preserves the open $\rho_{0}(H)$-orbit $\Omega_{0}$ in $X$ and induces a flow on the quotient manifold $\rho_{0}(\Gamma) \backslash \Omega_{0}$. The projection of $\Omega_{0}$ on $P(V)$ is contained in the complement of $L_{0}$, an affine plane. Consider the coordinate system $(u, v)$ on this plane such that the coordinates of $\left[u e_{1}+e_{2}+v e_{3}\right]$ are $(u, v)$.

Actually, the projection of $\Omega_{0}$ in $P(V)$ is the complement in this plane of the point $\left[e_{2}\right]$, ie, the complement of the origin $(u, v)=(0,0)$. The induced action of $\varphi^{t}$ on this projection is the homothety of factor $e^{t}$ fixing $\left[e_{2}\right]=(0,0)$.

On the other hand, if $h$ is an element of $H \approx \operatorname{SL}(2, \mathbb{R})$ of the form

$$
\left(\begin{array}{ll}
a & b \\
c & d
\end{array}\right)
$$

then $f_{+}(h)$ projects to $\left[\rho_{0}(h) e_{1}\right]$ in $P(V)$, with $(u, v)$-coordinates $(a, c)$. Hence, for any $t$, the $(u, v)$-coordinates of $f_{+}\left(h a^{t}\right)$ are $\left(e^{t} a, e^{t} c\right)$. Since the action of a transformation of $X$ is characterized by its projective action on $P(V)$, we see that, via the identification $\Omega_{0} \approx H$ we have selected, the action of $\varphi^{t}$ on $\Omega_{0}$ coincides with the right action of $a^{t}$ on $H$. Hence, the flow on $\rho_{0}(\Gamma) \backslash \Omega_{0}$ induced by $\varphi^{t}$ is conjugate to the flow $\left(M, \Phi^{t}\right)$

4.1.2 Linear deformations Let $u: \Gamma \rightarrow \mathbb{R}$ be any morphism. The horocyclic morphism can be deformed to a new morphism, called the linear $u$-deformation:

$$
\rho_{u}(\gamma)=\varphi^{u(\gamma)} \circ \rho_{0}(\gamma)
$$

The morphism $u$ is an element of $H^{1}(\Gamma, \mathbb{R})$. On this cohomology space, with $\rho_{0}: \Gamma \rightarrow H$ fixed, we can define the stable norm (cf [1]) as follows: for any hyperbolic element $\gamma$ of $\Gamma$, let $t(\gamma)$ be the double of the logarithm of $r(\gamma)$ (this is the length of the closed geodesic associated to $\Gamma$ in the quotient of the Poincare disc by $\Gamma$ ). For any element $\hat{\gamma}$ of $H_{1}(\Gamma, \mathbb{Z})$, and for any positive integer $n$, let $t_{n}(\hat{\gamma})$ be the infimum of the values $t(\gamma) / n$ where $\gamma$ describes all the elements of $\Gamma$ representing $n \hat{\gamma}$. The limit of $t_{n}(\hat{\gamma})$ exists; it is the stable norm of $\hat{\gamma}$ in $H_{1}(\Gamma, \mathbb{Z})$. This norm is extended

\footnotetext{
${ }^{2}$ The factor $1 / 3$ arises from the fact that this flow truly lies in PGL $(V)$ : multiplying every coefficient by the inverse of the middle diagonal coefficient provides a more elegant expression....
} 
in a unique way on all $H_{1}(\Gamma, \mathbb{R})$; the dual of it is the stable norm of $H^{1}(\Gamma, \mathbb{R})$. The stable norm of $u$ in $H^{1}(\Gamma, \mathbb{R})$ is denoted $|u|_{s}$.

Theorem 4.2 The representation $\rho_{u}$ is $(G, Y)-$ Anosov if and only if $|u|_{s}<1 / 2$.

Proof Assume that $\rho_{u}$ is $(G, Y)$-Anosov. The invariant curve $\Lambda$ must be the closure of the union of attractive fixed points of $\rho_{u}(\Gamma)$. In particular, it contains the closure of the attractive fixed points of the commutator subgroup $\rho_{u}([\Gamma, \Gamma])$. But $\rho_{0}$ and $\rho_{u}$ coincide on $[\Gamma, \Gamma]$, and attractive fixed points of elements of $[\Gamma, \Gamma]$ are dense in $\overline{\mathcal{S}}_{ \pm} \approx \mathbb{R} P^{1} ;$ hence, $\Lambda=\Lambda_{0}$.

But it is easy to see that if $|u|_{s}>1 / 2$, there is an element $\gamma$ of $\Gamma$ such that the attractive fixed point of $\rho_{u}(\gamma)$ in $P(V)$ is $\left[e_{2}\right]$. This attractive fixed point does not belong to $L$, which contradicts Corollary 3.14. Therefore, we must have $|u|_{s} \leq 1 / 2$. Since the $(G, Y)$-Anosov property is open in $\operatorname{Rep}(\Gamma, G)$, the inequality is strict: $|u|_{s}<1 / 2$.

The inverse statement, ie, the fact that $\rho_{u}$ is $(G, Y)$-Anosov if $|u|_{s}<1 / 2$, is proved in the Appendix.

Remark 4.3 As the proof above shows, the limit curve $\Lambda$ of $\rho_{u}$, when $|u|_{s}<1 / 2$, is the limit curve $\Lambda_{0}$ of $\rho_{0}$. It does not depend on the inclusion $\Gamma \subset H$.

\subsection{Deformations with $L_{0}$ remaining constant}

Consider morphisms $\rho: \Gamma \rightarrow G$ of the form

$$
\rho(\gamma)=\left(\begin{array}{ccc}
e^{u(\gamma) / 3} a(\gamma) & 0 & e^{u(\gamma) / 3} b(\gamma) \\
\mu(\gamma) & e^{-2 u(\gamma) / 3} & \nu(\gamma) \\
e^{u(\gamma) / 3} c(\gamma) & 0 & e^{u(\gamma) / 3} d(\gamma)
\end{array}\right)
$$

where:

- the representation

$$
\rho_{\lambda}: \gamma \mapsto\left(\begin{array}{cc}
a(\gamma) & b(\gamma) \\
c(\gamma) & d(\gamma)
\end{array}\right)
$$

is Fuchsian, taking value in $\operatorname{SL}(2, \mathbb{R})$, ie, is injective, with discrete image,

- $u: \Gamma \rightarrow \mathbb{R}$ is a morphism.

Such a representation is called a radial representation.

When $u$ has stable norm (relatively to $\rho_{\lambda}$ ) strictly less than $1 / 2, \rho$ is called a hyperbolic representation. In this case, $\left[e_{2}\right]$ is a fixed point of saddle type of every nontrivial $\rho(\gamma)$. 
In [2], the action of $\Gamma$ on $P(V)$ induced by such a representation is called a hyperbolic action.

One of the main results of [2] is:

Theorem 4.4 [2, Theorem A] Let $\rho$ be a hyperbolic representation. The action of $\rho(\Gamma)$ on $P(V)$ preserves a continuous closed simple curve $L$. This curve, if Lipschitz, is a projective line.

Actually, the first part of this Theorem is a corollary of the following:

Proposition 4.5 Hyperbolic representations are $(G, Y)$-Anosov.

Proof If the maps $\mu$ and $v$ are trivial, it follows from Theorem 4.2. Then, observe that the composition of a hyperbolic representation by the conjugacy in $G$ by $\varphi^{t}$ remains hyperbolic radial, with the same $\rho_{\lambda}$ and the same $u$, but with coefficients $\mu$, $v$ multiplied by $e^{-2 t}$. Since $\rho_{u}$ is $(G, Y)$-Anosov, and by stability of $(G, Y)$-Anosov representations, the conjugated representation for big $t$ is $(G, Y)$-Anosov. The lemma follows since conjugacy in $G$ does not affect the $(G, Y)$-Anosov property.

Remark 4.6 The invariant curve $L^{*}$ in $P\left(V^{*}\right)$ is obviously $L_{0}^{*}=\left[e_{2}^{\perp}\right]$.

As a corollary, we get the proof of Theorem 1.1, that we restate here for reader's convenience:

Theorem 1.1 Hyperbolic representations are $(G, Y)$-Anosov. More precisely, a radial representation is $(G, Y)$-Anosov if and only if it is hyperbolic.

Proof The first part is Proposition 4.5. The proof of the second assertion is similar to the proof of Theorem 4.2 and is left to the reader.

Proposition 4.7 Let $\rho: \Gamma \rightarrow G$ be a $(G, Y)-$ Anosov representation. Assume that a finite index subgroup of $\rho(\Gamma)$ preserves a proper subspace of $V$. Then, up to conjugacy in $G, \rho$ or $\rho^{*}$ is a hyperbolic representation.

Proof A proper subspace of $V$ is a line or a 2-plane: replacing $\rho$ by $\rho^{*}$ if necessary, we can assume that the subspace preserved by a finite index subgroup of $\rho(\Gamma)$ is a line. After conjugacy in $G$, we can assume moreover that this invariant line is spanned by $e_{2}$. Let $\Gamma^{\prime} \subset \Gamma$ be the finite index subgroup such that $\rho\left(\Gamma^{\prime}\right)$ fixes $\left[e_{2}\right]$. The restriction $\rho^{\prime}$ of $\rho$ to $\Gamma^{\prime}$ is still $(G, Y)-$ Anosov, and its limit curve $\Lambda^{\prime}$ is the limit curve $\Lambda$ of $\rho$. 
According to Proposition 3.2, $\rho^{\prime}$ is faithful, with discrete image. It follows that $\rho^{\prime}$ is a radial representation, described as above by a Fuchsian representation $\rho_{\lambda}^{\prime}: \Gamma^{\prime} \rightarrow H$ and a morphism $u^{\prime}: \Gamma \rightarrow \mathbb{R}$. This morphism is trivial on $\left[\Gamma^{\prime}, \Gamma^{\prime}\right]$. It follows that $\left[e_{2}\right]$ is a saddle fixed point of $\rho^{\prime}(\gamma)$ for every element $\gamma$ of $\left[\Gamma^{\prime}, \Gamma^{\prime}\right]$. Hence, the attractive fixed point of $\left(\rho^{\prime}\right)^{*}(\gamma)$ in $P\left(V^{*}\right)$ belongs to $\left[e_{2}^{\perp}\right]$. The argument used in the proof of Theorem 4.2 and the identity $\Lambda=\Lambda^{\prime}$ imply here that the invariant curve $L_{0}^{*}$ is $\left[e_{2}^{\perp}\right]$. In particular, it follows that $\left[e_{2}\right]$ is a saddle fixed point of $\rho(\gamma)$ for every $\gamma$ in $\Gamma$, not only in $\left[\Gamma^{\prime}, \Gamma^{\prime}\right]$. Therefore, $\rho$ is a linear $u$-deformation of a horocyclic representation. As in the proof of Theorem 4.2, we infer $|u|_{s} \leq 1 / 2$, and, finally, $|u|_{s}<1 / 2$ (by stability of Anosov representations).

\section{Properness of the action on $\Omega$}

Let $\rho: \Gamma \rightarrow G$ be a $(G, Y)$-Anosov representation. Recall that $\rho(\Gamma)$ preserves the open domain $\Omega$, which is the set of flags $\left([u],\left[u^{*}\right]\right)$ with $[u] \notin L,\left[u^{*}\right] \notin L^{*}$. The main task of this section is to prove Theorem 1.2, ie that the action of $\rho(\Gamma)$ on $\Omega$ is proper.

The proof will follow from Proposition 5.6 below. In order to state this proposition, we need to introduce some notation.

Definition 5.1 A sequence $\left(\gamma_{n}\right)_{(n \in \mathbb{N})}$ in $\Gamma$ is unbounded if the sequence $\rho\left(\gamma_{n}\right)_{(n \in \mathbb{N})}$ escapes from any compact of $G$.

Let $\left(\gamma_{n}\right)_{(n \in \mathbb{N})}$ be an unbounded sequence in $\Gamma$. We denote $g_{n}=\rho\left(\gamma_{n}\right)$ and $g_{n}^{*}$ the dual of $g_{n}$ in $\operatorname{SL}(3, \mathbb{R})$ (cf Section 2.1).

Remember that we equip $V, V^{*}$ with the Euclidean metrics $N, N^{*}$ for which $\left(\left[e_{1}\right],\left[e_{2}\right],\left[e_{3}\right]\right)$ and $\left(\left[e_{1}^{*}\right],\left[e_{2}^{*}\right],\left[e_{3}^{*}\right]\right)$ are an orthonormal basis. It induces a norm on $\mathfrak{g l}(V)$ : the operator norm $\|$. Let denote $\bar{g}_{n}, \bar{g}_{n}^{*}$ the quotients $g_{n} /\left\|g_{n}\right\|$ and $g_{n}^{*} /\left\|g_{n}^{*}\right\|$. Observe that the actions of $g_{n}$ and $\bar{g}_{n}$ on $P(V)$ coincide, as for the actions of $g_{n}^{*}$ and $\bar{g}_{n}^{*}$ on $P\left(V^{*}\right)$.

Definition 5.2 The unbounded sequence $\left(\gamma_{n}\right)_{(n \in \mathbb{N})}$ is converging if the sequences $\left(\bar{g}_{n}\right)_{(n \in \mathbb{N})}$ and $\left(\bar{g}_{n}^{*}\right)_{(n \in \mathbb{N})}$ both converge.

The quotients $\bar{g}_{n}$ and $\bar{g}_{n}^{*}$ belong to the unit sphere of $\|$, which is compact. Therefore:

Lemma 5.3 Every unbounded sequence admits converging subsequences. 
Assume now that $\left(\gamma_{n}\right)_{(n \in \mathbb{N})}$ is converging. Denote by $\bar{g}, \bar{g}^{*}$ the limits of $\left(\bar{g}_{n}\right)_{(n \in \mathbb{N})}$ and $\left(\bar{g}_{n}^{*}\right)_{(n \in \mathbb{N})}$. Let $I$ be the image of $\bar{g}, I_{*}$ the image of $\bar{g}^{*}, K$ the kernel of $\bar{g}$ and $K_{*}$ the kernel of $\bar{g}^{*}$. By convention, $[I],[K],\left[I_{*}\right]$ and $\left[K_{*}\right]$ are their projections in $P(V), P\left(V^{*}\right)$. Let $I^{\perp}, K^{\perp} \subset V^{*}$ and $K_{*}^{\perp}, I_{*}^{\perp} \subset V$ be the dual subspaces, and $\left[I^{\perp}\right],\left[K^{\perp}\right], \ldots$ their respective projectivizations.

Lemma 5.4 The sequence $\left(\bar{g}_{n}\right)_{(n \in \mathbb{N})}$ converge uniformly on compact subsets of $P(V) \backslash[K]$ to the restriction of $\bar{g}$ to $P(V) \backslash[K]$. Similarly, the sequence $\left(\bar{g}_{n}^{*}\right)_{(n \in \mathbb{N})}$ converge uniformly on compact subsets of $P\left(V^{*}\right) \backslash\left[K_{*}\right]$ to the restriction of $\bar{g}$.

Remark 5.5 The image of the restriction of $\bar{g}$ to $P(V) \backslash[K]$ is $[I]$, and the image of the restriction of $\bar{g}^{*}$ to $P\left(V^{*}\right) \backslash\left[K_{*}\right]$ is $\left[I_{*}\right]$.

We can now state the main result of this section:

Proposition 5.6 For any converging unbounded sequence, $I$ and $I^{*}$ have dimension 1 (and therefore, $K$ and $K^{*}$ have dimension 2). Moreover, the points $[I],\left[K_{*}^{\perp}\right]$ belong to $L$, and $\left[I_{*}\right],\left[K^{\perp}\right]$ belong to $L^{*}$.

Consider the Cartan decompositions of $g_{n}, g_{n}^{*}$ according to the canonical basis

$$
\begin{aligned}
& g_{n}=k_{n}\left(\begin{array}{ccc}
\lambda_{n} & 0 & 0 \\
0 & \mu_{n} & 0 \\
0 & 0 & v_{n}
\end{array}\right) l_{n}^{-1} \\
& g_{n}^{*}=k_{n}\left(\begin{array}{ccc}
\lambda_{n}^{-1} & 0 & 0 \\
0 & \mu_{n}^{-1} & 0 \\
0 & 0 & v_{n}^{-1}
\end{array}\right) l_{n}^{-1}
\end{aligned}
$$

where $k_{n}, l_{n}$ are isometries of $N$, and $\lambda_{n} \geq \mu_{n} \geq v_{n}$, with $\lambda_{n} \mu_{n} v_{n}=1$.

Up to a subsequence, we can assume without loss of generality that all the $\bar{k}_{n}$ and $\bar{l}_{n}$ admits limits $\bar{k}$ and $\bar{l}$. Then, the matrices $\bar{g}$ and $\bar{g}^{*}$ have the expressions:

$$
\begin{aligned}
\bar{g} & =\bar{k}\left(\begin{array}{ccc}
\bar{\lambda} & 0 & 0 \\
0 & \bar{\mu} & 0 \\
0 & 0 & 0
\end{array}\right) \bar{l}^{-1} \\
\bar{g}^{*} & =\bar{k}\left(\begin{array}{ccc}
0 & 0 & 0 \\
0 & \bar{\mu}^{\prime} & 0 \\
0 & 0 & \bar{v}
\end{array}\right) \bar{l}^{-1}
\end{aligned}
$$

where $\bar{\lambda}, \bar{v}$ are positive real numbers, $\bar{\mu}, \bar{\mu}^{\prime}$ are nonnegative real numbers. 
The $g_{n}$ all have determinant 1 . Hence, since the sequence is unbounded, the norms $\left\|g_{n}\right\|$ and $\left\|g_{n}^{*}\right\|$ tend to $+\infty$. If $\bar{g}^{t}$ denotes the transpose matrix of $\bar{g}$, we have:

$$
\bar{g}^{t} \circ \bar{g}^{*}=0=\bar{g}^{*} \circ \bar{g}^{t}
$$

Therefore we must have $\bar{\mu} \bar{\mu}^{\prime}=0$. In other words:

$$
I_{*} \subset I^{\perp}, \quad K^{\perp} \subset K_{*}
$$

We will also be useful to consider the inverse sequence $\left(\gamma_{n}^{-1}\right)_{(n \in \mathbb{N})}$. This amounts to replacing $g_{n}$ and $g_{n}^{*}$ by their inverse $h_{n}, h_{n}^{*}$ :

$$
\begin{aligned}
& h_{n}=l_{n}\left(\begin{array}{ccc}
\lambda_{n}^{-1} & 0 & 0 \\
0 & \mu_{n}^{-1} & 0 \\
0 & 0 & v_{n}^{-1}
\end{array}\right) k_{n}^{-1} \\
& h_{n}^{*}=l_{n}\left(\begin{array}{ccc}
\lambda_{n} & 0 & 0 \\
0 & \mu_{n} & 0 \\
0 & 0 & v_{n}
\end{array}\right) k_{n}^{-1}
\end{aligned}
$$

Their limits for $n \rightarrow+\infty$ are:

$$
\begin{aligned}
\bar{h} & =\bar{l}\left(\begin{array}{ccc}
0 & 0 & 0 \\
0 & \bar{\mu}^{\prime} & 0 \\
0 & 0 & \bar{v}
\end{array}\right) \bar{k}^{-1} \\
\bar{h}^{*} & =\bar{l}\left(\begin{array}{ccc}
\bar{\lambda} & 0 & 0 \\
0 & \bar{\mu} & 0 \\
0 & 0 & 0
\end{array}\right) \bar{k}^{-1}
\end{aligned}
$$

Hence:

Lemma 5.7 The image of $\bar{h}$ and $\bar{h}^{*}$ are respectively $K_{*}^{\perp}, K^{\perp}$. Their kernel are $I_{*}^{\perp}$, $I^{\perp}$, respectively.

Lemma 5.8 The image by $\bar{g}$ of $L \backslash[K]$ is contained in $L \cap[I]$. Similarly:

$$
\bar{g}^{*}\left(L^{*} \backslash\left[K^{*}\right]\right) \subset L^{*} \cap\left[I^{*}\right]
$$

Proof This is a direct corollary of the $\Gamma$-invariance of $L, L^{*}$.

Lemma 5.9 If $I$ has dimension one, then, $[I]$ lies in $L$. Similarly, if $I^{*}$ has dimension one, then $\left[I^{*}\right]$ belongs to $L^{*}$. 
Proof Assume a contrario that $[I]$ is a single point which is not in $L$. Then, according to Lemma 5.8, $L \backslash[K]$ is empty since its image by $\bar{g}$ is empty. Hence $L$ is contained in $[K]$, and since $L$ and $[K]$ are simple curves, we have $L=[K]$. In particular, $[I]$ does not belong to $[K]$. Let $D$ be a small open disk containing $[I]$, but with closure disjoint from $[K]=L$. By Lemma 5.4, for $n$ sufficiently big, the image by $g_{n}$ of the closure of $D$ is contained in $D$. Hence, $D$ contains the attractive fixed point of some $g_{n}$. Taking arbitrarily small $D$, we obtain that $[I]$ is the limit of a sequence of attractive fixed points of elements of $\rho(\Gamma)$. By Corollary 3.14, $[I]$ belongs to $L$. Contradiction.

The dual case $\operatorname{dim}\left(I^{*}\right)=1$ is similar.

Lemma 5.10 If $I$ (respectively $I^{*}$ ) has dimension one, then $\left[K^{\perp}\right]$ lies in $L^{*}$ (respectively $\left[K_{*}^{\perp}\right]$ lies in $\left.L\right)$.

Proof If $I$ has dimension one, then the image $K^{\perp}$ of $\bar{h}^{*}$ has dimension one (cf Lemma 5.7). Apply then Lemma 5.9 to the inverse sequence.

We are now ready to prove Proposition 5.6:

Proof of Proposition 5.6 Assume that $I$ has dimension two. Then $I^{\perp}$ has dimension one, and the inclusion $I_{*} \subset I^{\perp}$ is actually an equality. Hence, according to Lemma 5.9 the limit curve $L^{*}$ contains the point $\left[I_{*}\right]$. According to Corollary 3.12, the intersection $L \cap[I]=L \cap\left[I_{*}^{\perp}\right]$ is a single point. Hence, by Lemma 5.8, the image $\bar{g}(L \backslash[K])$ is reduced to a single point. But the preimage by $\bar{g}$ of any point in $[I]$ is $d \backslash[K]$, where $d$ is a projective line containing the single point $[K]$. It follows that $L$ is a projective line.

Dually, since the image $\left[K^{\perp}\right]$ of $\bar{h}^{*}$ has dimension two, the same argument applied to the inverse sequence proves that $L^{*}$ is a projective line. It follows that $\rho$ is actually the $u$-deformation of a horocyclic representation

where

$$
\begin{aligned}
& \rho(\gamma)=\left(\begin{array}{ccc}
e^{u(\gamma) / 3} a(\gamma) & 0 & e^{u(\gamma) / 3} b(\gamma) \\
0 & e^{-2 u(\gamma) / 3} & 0 \\
e^{u(\gamma) / 3} c(\gamma) & 0 & e^{u(\gamma) / 3} d(\gamma)
\end{array}\right) \\
& \rho_{\lambda}: \gamma \mapsto\left(\begin{array}{cc}
a(\gamma) & b(\gamma) \\
c(\gamma) & d(\gamma)
\end{array}\right)
\end{aligned}
$$

is a Fuchsian morphism into $\operatorname{SL}(2, \mathbb{R})$, and $u: \Gamma \rightarrow \mathbb{R}$ a morphism. According to Proposition 4.7, the stable norm of $u$ satisfies $|u|_{s}<1 / 2$. 
Let $l(\gamma)$ be as in [16] the logarithm of the spectral radius of the diagonal matrix appearing in the Cartan decomposition of $\gamma$ in $\operatorname{SL}(2, \mathbb{R})$. Then, the logarithms of the coefficients $\lambda_{n}, \mu_{n}$ and $v_{n}$ are, modulo some common additive constant, the quantities $u\left(\gamma_{n}\right)+l\left(\gamma_{n}\right), u\left(\gamma_{n}\right)$ and $u\left(\gamma_{n}\right)-l\left(\gamma_{n}\right)$. In the proof of Théorème 3.4 in [16], it is shown that $l\left(\gamma_{n}\right)-\left|u\left(\gamma_{n}\right)\right|$ tends to $+\infty$ : it precisely means $\bar{\mu}=0$, ie that $I$ has dimension one. Contradiction.

This contradiction proves that $I$ has dimension one. Similarly, $I^{*}$ has dimension one. The other assertions of Proposition 5.6 are then corollaries of Lemmas 5.9 and 5.10.

Proof of Theorem 1.2 Assume that the action of $\rho(\Gamma)$ on $\Omega$ is not proper. Since $\rho(\Gamma)$ is discrete, there is an unbounded sequence $\left(\gamma_{n}\right)_{(n \in \mathbb{N})}$ in $\Gamma$ and a sequence of flags $\left(\left[u_{n}\right],\left[u_{n}^{*}\right]\right)_{(n \in \mathbb{N})}$ in $\Omega$ satisfying:

(1) the sequence $\left(\left[u_{n}\right],\left[u_{n}^{*}\right]\right)_{(n \in \mathbb{N})}$ converge to a point $\left([\bar{u}],\left[\bar{u}^{*}\right]\right)$ in $\Omega$,

(2) the sequence $\rho\left(\gamma_{n}\right)\left(\left[u_{n}\right],\left[u_{n}^{*}\right]\right)_{(n \in \mathbb{N})}=\left(\left[v_{n}\right],\left[v_{n}^{*}\right]\right)_{(n \in \mathbb{N})}$ also converges to a flag $\left([\bar{v}],\left[\bar{v}^{*}\right]\right)$ in $\Omega$.

Without loss of generality, we can assume that $\left(\gamma_{n}\right)_{(n \in \mathbb{N})}$ is converging. Since $\left([\bar{v}],\left[\bar{v}^{*}\right]\right)$ belongs to $\Omega,[\bar{v}]$ does not belong to $L$. According to Proposition 5.6, $\bar{v} \neq[I]$. It follows, with Lemma 5.4 , that $[\bar{u}]$ belongs to $[K]$. Similarly, we have $\left[\bar{u}^{*}\right] \in\left[K_{*}\right]$. Hence:

- the projective line $\left[K_{*}\right]$ in $P\left(V^{*}\right)$ contains $\left[\bar{u}^{*}\right]$ and $\left[K^{\perp}\right]$ (since $K^{\perp} \subset K_{*}$ ),

- the projective line $\left[\bar{u}^{\perp}\right]$ in $P\left(V^{*}\right)$ contains $\left[\bar{u}^{*}\right]$ (since $\left.\left\langle\bar{u} \mid \bar{u}^{*}\right\rangle=0\right)$ and $\left[K^{\perp}\right]$ (since $[\bar{u}] \in[K])$.

Since $\left[\bar{u}^{*}\right]$ does not belong to $L^{*}$, and since by Proposition 5.6 the single point $\left[K^{\perp}\right]$ belongs to $L^{*}$, we have $\bar{u}^{*} \neq\left[K^{\perp}\right]$. Hence the projective lines $\left[K_{*}\right]$ and $\left[\bar{u}^{\perp}\right]$ share two distinct points: they are equal. In other words, $[\bar{u}]$ is equal to $\left[K_{*}^{\perp}\right]$ : according to Proposition 5.6, it belongs to $L$, a contradiction.

Remark 5.11 Theorem 1.2 is true for any $(G, Y)$-Anosov representations, in particular, for representations in the Hitchin component, ie, dividing a strictly convex domain $C$ of $P(V)$. In this case, we recover the well-known properness of the action on the projectivized tangent bundle of $C$. But we also obtain the properness of the action on "timelike directions" over the "de Sitter-like" component $P(V) \backslash \bar{C}$.

Remark 5.12 We can ask how Theorem 1.2 can be extended to any other $(G, Y)-$ Anosov representations, for other pairs $(G, Y)$. For example, for the pair $(G, Y)=$ (PSL $(2, \mathbb{C}), \mathbb{C} P^{1} \times \mathbb{C} P^{1} \backslash$ diag), ie, the case of conformal quasi-Fuchsian representations, Theorem 1.2 corresponds the well-known properness of the action on the discontinuity domain, ie, the complement in $\mathbb{C} P^{1}$ of the limit set. 
Remark 5.13 In the wonderful paper [17], R Schwarz considers some particular actions of $\operatorname{PSL}(2, \mathbb{Z})$ on the flag manifold $X$. He exhibited invariant curves $L, L^{*}$ with properties completely similar to the invariant curves $L, L^{*}$ we have considered here ${ }^{3}$ : compare our Corollaries 3.12 and 3.13 with Theorem 3.3 of [17]. Our Theorem 1.2 echoes Theorem 4.2 of [17]. Hence, it seems reasonable to qualify these representations of $\operatorname{PSL}(2, \mathbb{Z})$ as $(G, Y)$-Anosov representations. In fact, they are also deformations of the representation obtained by composing $\rho_{0}: H \rightarrow G$ with the inclusion $\operatorname{SL}(2, \mathbb{Z}) \subset$ $\operatorname{SL}(2, \mathbb{R})$. But there is a crucial difference: $\operatorname{PSL}(2, \mathbb{Z})$ is indeed a lattice of $\operatorname{PSL}(2, \mathbb{R})$, but not cocompact! It is therefore presumably possible and interesting to extend the notion of $(G, Y)$-Anosov representations to nonuniform lattices of $H$.

\section{Anosov flag manifolds}

Thanks to Theorem 1.2, Definition 2.15 can be extended:

Definition 6.1 Let $\rho: \Gamma \rightarrow G$ be a $(G, Y)$-Anosov representation. The quotient by $\rho(\Gamma)$ of the domain $\Omega$ is called an Anosov flag manifold.

\subsection{Tautological foliations}

The fibers of the projections $X \rightarrow P(V), P\left(V^{*}\right)$ are leaves of foliations by circles on $X$. They are preserved by $G$; therefore, they induce two 1 -dimensional foliations on any flag manifold. The foliation corresponding to the projection $X \rightarrow P(V)$ is called the first tautological foliation. The other one, corresponding to the projection $X \rightarrow P\left(V^{*}\right)$, is called the second tautological foliation. Observe that these foliations are both transversely real projective.

Remark 6.2 When $\rho$ is hyperconvex, $\Omega$ has three connected components (see Remark 3.17):

- One is the set of flags $\left([v],\left[v^{*}\right]\right)$ with $[v] \in[C]$. We denote it by $\Omega_{1}$. The quotient $M_{1}=\rho(\Gamma) \backslash \Omega_{1}$ is naturally identified with the projectivized tangent bundle of the convex real projective surface $S=\rho(\Gamma) \backslash[C]$. The leaves of the first tautological foliation are the fibers of the bundle map. In particular, they are compact. The second tautological foliation is the foliation supported by the geodesic flow of the Hilbert metric on $S$, quotiented by the involution sending any tangent vector to its opposite: hence, it is topologically conjugate to the geodesic flow of any hyperbolic metric on

\footnotetext{
${ }^{3}$ There is a minor difference: the action on $X$ considered in [17] contains polarities, ie, projective transformations followed by the flip $\left([v],\left[v_{*}\right]\right) \mapsto\left(\left[v_{*}^{\perp}\right],\left[v^{\perp}\right]\right)$.
} 
the surface $\Sigma$ quotiented by the antipodal map in the fibers (see our paper [2] for more details).

- Another component, $\Omega_{2}$, which is the set of flags $\left([v],\left[v^{*}\right]\right)$ with $\left[v^{*}\right] \in\left[C^{*}\right]$. The quotient $M_{2}=\rho(\Gamma) \backslash \Omega_{2}$ is the projectivized tangent bundle of the dual convex real projective surface $S^{*}=\rho^{*}(\Gamma) \backslash\left[C^{*}\right]$. The leaves of the second tautological foliation are the fibers of the bundle map, whereas the first tautological foliation is doubly covered by the geodesic flow on $\Sigma$.

- The third component, $\Omega_{3}$, is the "de Sitter"-like component. It is the set of pairs $\left([v],\left[v^{*}\right]\right)$, where $[v]$ belongs to the Möbius band $\mathcal{A}$, which is the complement in $P(V)$ of the closure of $[C]$, and $\left[v^{*}\right]$ belongs to the Möbius band $\mathcal{A}^{*}$, which is the complement in $P\left(V^{*}\right)$ of the closure of $\left[C^{*}\right]$. Observe that for any $[v]$ in $\mathcal{A}$, the intersection $\left[v^{\perp}\right] \cap L^{*}$ is the union of two points $\left[v_{-}^{*}\right],\left[v_{+}^{*}\right]$ (note that $L^{*}=\partial\left[C^{*}\right]$ ). Then, $\left[v_{ \pm}^{*}\right]$ is tangent to $L=\partial[C]$ at a point $\left[v_{ \pm}\right]$. Let $p([v])$ be the intersection of $\left[\left(v^{*}\right)^{\perp}\right]$ and the projective line $\left[w^{*}\right]$ containing $\left[v_{-}\right]$and $\left[v_{+}\right]$; it belongs to $[C]$. Hence, the flag $\left(p([v]),\left[w^{*}\right]\right)$ belongs to $\Omega_{1}$. We have thus defined a $\rho(\Gamma)$-equivariant map from $\Omega_{3}$ into $\Omega_{1}$. It is easy to show that it is a homeomorphism, and that it sends the first tautological foliation of $\Omega_{3}$ onto the second tautological foliation of $\Omega_{1}$.

A similar treatment can be applied to the second tautological foliation on $\Omega_{3}$, leading to the following statement: the tautological foliations of $M_{3}=\rho(\Gamma) \backslash \Omega_{3}$ are both topologically conjugate to the geodesic flow of any hyperbolic metric on the surface $\Sigma$ quotiented by the antipodal map in the fibers.

\subsection{Canonical Goldman flag manifolds}

In [2], Anosov flag manifolds associated to linear $u$-deformations of horocyclic representations were defined and called canonical Goldman flag manifolds.

We recall that in the case of canonical Goldman flag manifolds, the flow $\varphi^{t}$ induces an Anosov flow on the associated flag manifold. More precisely, this flow is a double covering of a Desarguian Anosov flow as defined in [9; 3]. The tautological foliations are then the strong stable and unstable foliations. More details can be found in [2].

For our purpose here, it is more suitable to drop the identification $G=\operatorname{PGL}(V) \approx$ $\operatorname{SL}(V)$, and to consider $u$-deformations as morphisms $\lambda: \Gamma \rightarrow \mathrm{GL}_{2}$, where $\mathrm{GL}_{2}$ is the group of invertible $2 \times 2$ matrices, identified with the stabilizer in $G$ of $\left[\left(L_{0}^{*}\right)^{\perp}\right]=\left[e_{2}\right]$ and $L_{0}=\left[\left(e_{2}^{*}\right)^{\perp}\right]$ :

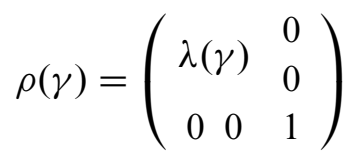


The group $\mathrm{GL}_{2}$ preserves the annulus $A_{\text {can }}=P(V) \backslash\left(L_{0} \cup\left[e_{2}\right]\right)$ (there is also a dual action on the annulus $P\left(V^{*}\right) \backslash\left(L_{0}^{*} \cup\left[e_{2}^{*}\right]\right)$. The minimality and unique ergodicity of horocyclic flows [5] imply that the $\lambda(\Gamma)$-action on $A_{\text {can }}$ is minimal and uniquely ergodic: up to a positive factor, there is one and only Borel measure on $A_{\text {can }}$ preserved by $\lambda(\Gamma)$.

Proposition 6.3 Two inclusions $\lambda_{1}, \lambda_{2}$ from a surface group $\Gamma$ into $\mathrm{GL}_{2}$ induce conjugate actions on the annulus $A_{\text {can }}$ if and only if $\lambda_{1}, \lambda_{2}$ are conjugate in $\mathrm{GL}_{2}$.

Proof This is a folkloric fact, but we don't know any appropriate reference.

One of the implication is clear. Let's prove the inverse statement: let $f: A_{\text {can }} \rightarrow A_{\text {can }}$ be a homeomorphism conjugating the actions of $\Gamma$ on $A_{\text {can }}$ via $\lambda_{1}, \lambda_{2}$, respectively. Clearly, $f$ extends to a homeomorphism $f: P(V) \backslash L_{0} \rightarrow P(V) \backslash L_{0}$, still $\Gamma-$ equivariant. Consider $\varphi^{t}$ as a group of projective transformations of $A_{\text {can }}$.

For any nontrivial $\gamma$ in $\Gamma$, $\left[e_{2}\right]$ is saddle fixed point of $\lambda_{1}(\gamma)$ and $\lambda_{2}(\gamma)$, and the $\lambda_{1}(\gamma)-$ or $\lambda_{2}(\gamma)$-stable leaf of $\left[e_{2}\right]$ is the union of two orbits of $\varphi^{t}$, with $\left[e_{2}\right]$ itself. Moreover, when $\gamma$ is varying, these stable leaves form a dense subset among the orbits of $\varphi^{t}$. It follows that $f$ sends $\varphi^{t}$-orbits on $\varphi^{t}$-orbits. Hence, there is a continuous function $\alpha: \mathbb{R} \times A_{\text {can }} \rightarrow \mathbb{R}$ such that:

$$
f\left(\varphi^{t}([u])\right)=\varphi^{\alpha(t,[u])} f([u])
$$

For a fixed $t$, since $\varphi^{t}$ commutes with the $\Gamma$-actions, $[u] \mapsto \alpha(t,[u])$ is $\lambda_{1}(\Gamma)$-invariant. Since the action of $\lambda_{1}(\Gamma)$ on the annulus is minimal, it follows that $\alpha$ depends only on $t$. Since $f^{-1} \circ \varphi^{t} \circ f$ is a one-parameter subgroup of $G, \alpha: \mathbb{R} \rightarrow \mathbb{R}$ is a morphism: there is a positive constant $C$ such that $\alpha(t)=C t$.

For some nontrivial $\gamma$ in $\Gamma$, and for $j=1,2$, denote by $\pm e^{a_{j}(\gamma)}, \pm e^{b_{j}(\gamma)}$ the eigenvalues $\neq 1$ of $\lambda_{j}(\gamma)$, with $b<0<a$. Then, the $\lambda_{j}(\gamma)$-stable leaf of $\left[e_{2}\right]$ is the fixed point set of $\lambda_{1}(\gamma) \varphi^{-b_{1}(\gamma)}$. Its image by $f$ is the fixed point set of $\lambda_{2}(\gamma) \varphi^{-C b_{1}(\gamma)}$. It follows that $b_{2}(\gamma)=C b_{1}(\gamma)$. Similarly, $a_{2}(\gamma)=C a_{1}(\gamma)$.

Consider the projections of $\lambda_{j}(\gamma)(\Gamma)$ in $\operatorname{PSL}(2, \mathbb{R})=\bar{H}$. They are Fuchsian subgroups, corresponding to metrics $g_{1}, g_{2}$ on the surface $\Sigma$ with constant curvature -1 . Then, $a_{2}(\gamma)=C a_{1}(\gamma)$ and $b_{2}(\gamma)=C b_{1}(\gamma)$ imply that the $g_{2}$-length of a closed geodesic $c$ is $C$ times the $g_{1}$-length of the closed geodesic freely homotopic to $c$. This is possible only if $C=1$ (see for example Fathi, Laudenbach and Poenaru [8]; on page 137, end of "Exposé 7", it is proved that the hypothesis $(\mathrm{H})$ on page 136 , ie, the claim $C \neq 1$, is impossible). 
Therefore, if $\operatorname{Tr}$ denotes the trace function on the algebra $\mathfrak{g l}_{2}$ of $2 \times 2$ matrices:

$$
\operatorname{Tr} \circ \lambda_{1}=\operatorname{Tr} \circ \lambda_{2}
$$

It is well-known that this implies that $\lambda_{1}$ and $\lambda_{2}$ are conjugate in $\mathrm{GL}_{2}$. Let's recall the argument: since $\lambda_{j}$ are irreducible representations, every element $g$ of $\mathfrak{g l}_{2}$ can be (nonuniquely) written as a sum $\sum_{i} \eta_{i} \lambda_{j}\left(\gamma_{i}\right)$.

For every $g$ in $\mathfrak{g l}_{2}$, select such a decomposition $g=\sum_{i} \eta_{i} \lambda_{1}\left(\gamma_{i}\right)$. Then define $\phi(g)=\sum_{i} \eta_{i} \lambda_{2}\left(\gamma_{i}\right)$. The key point is that $\phi(g)$ does not depend on the selected decomposition of $g$. Indeed, if $0=\sum_{i} \eta_{i} \lambda_{1}\left(\gamma_{i}\right)$, then, for every $g^{\prime}=\sum_{k} v_{k} \lambda_{2}\left(\gamma_{k}\right)$ in $\mathfrak{g l}_{2}$, we have:

$$
\begin{aligned}
\operatorname{Tr}\left(\left(\sum_{i} \eta_{i} \lambda_{2}\left(\gamma_{i}\right)\right) g^{\prime}\right) & =\operatorname{Tr}\left(\sum_{i, k} \eta_{i} v_{k} \lambda_{2}\left(\gamma_{i}\right) \lambda_{2}\left(\gamma_{k}\right)\right) \\
& =\sum_{i, k} \eta_{i} v_{k} \operatorname{Tr}\left(\lambda_{2}\left(\gamma_{i} \gamma_{k}\right)\right) \\
& =\sum_{i, k} \eta_{i} v_{k} \operatorname{Tr}\left(\lambda_{1}\left(\gamma_{i} \gamma_{k}\right)\right) \\
& =\operatorname{Tr}\left(\left(\sum_{i} \eta_{i} \lambda_{1}\left(\gamma_{i}\right)\right)\left(\sum_{k} v_{k} \lambda_{1}\left(\gamma_{k}\right)\right)\right)=0
\end{aligned}
$$

Since this holds for every $g^{\prime}$, and since $g^{\prime} \mapsto \operatorname{Tr}\left(g_{0} g^{\prime}\right)$ can be zero only if $g_{0}=0$, we obtain that $0=\sum_{i} \eta_{i} \lambda_{2}\left(\gamma_{i}\right)$. As a corollary, $\phi$ is well-defined.

This map $\phi$ is obviously an algebra automorphism of $\mathfrak{g l}_{2}$; but such an automorphism is known to be an inner automorphism. Moreover, $\phi \circ \lambda_{1}=\lambda_{2}$. The proposition follows.

\subsection{Goldman manifolds}

In [2] we defined Goldman flag manifolds as Anosov flag manifolds associated to hyperbolic representations, ie $(G, X)$-Anosov representations for which $L^{*}$ is a projective line.

Let $\rho: \Gamma \rightarrow G$ be a hyperbolic representation. We can assume that, in the notation of the beginning of Section 4.2, $\rho_{0}(\gamma)=e^{u(\gamma)} \rho_{\lambda}$.

Proposition 6.4 The actions of $\rho(\Gamma)$ and $\rho_{0}(\Gamma)$ on $P(V)$ are topologically conjugate. 
Proof We reproduce the short proof in [2]: there is a (Hölder) continuous and homogeneous degree one map $\delta: \mathbb{R}^{2} \rightarrow \mathbb{R}$ such that $\left[x e_{1}+y e_{3}+z e_{2}\right]$ belongs to the invariant curve $L$ if and only if $z=\delta(x, y)$. The $\rho(\gamma)$-invariance of $L$ implies

$$
\delta(\rho(\gamma))=\delta(\gamma)+\tau_{1}(\gamma) x+\tau_{2}(\gamma) y
$$

where $\tau_{1}(\gamma)=e^{u(\gamma)} \mu(\gamma), \tau_{2}(\gamma)=e^{u(\gamma)} v(\gamma)$ (for $\mu(\gamma), v(\gamma)$ as in Section 4.2). It follows immediately that $(x, y, z) \mapsto(x, y, z+\delta(x, y))$ induces on $P(V)$ the required topological conjugacy between $\rho_{0}(\Gamma)$ and $\rho(\Gamma)$.

This proposition implies Theorem 5.1 of [2]: the first tautological foliation on a Goldman flag manifold is topologically conjugate to the horocyclic flow of a Desarguian Anosov flow. It follows also that the action of $\rho(\Gamma)$ on $P(V) \backslash\left(L \cup\left[e_{2}\right]\right)$ is minimal and uniquely ergodic.

\subsection{Nonhyperconvex Anosov flag manifolds}

Anosov flag manifolds with hyperconvex (ie, quasi-Fuchsian) representations are fairly well understood (see Remarks 3.17 and 5.11). From now, we exclude this case.

Lemma 6.5 The complements $P(V) \backslash L$ and $P\left(V^{*}\right) \backslash L^{*}$ are topological discs.

Proof Assume that $L^{*}$ is a projective line, ie, that $\rho$ is a hyperbolic representation: consider an affine domain $U \subset P(V)$ not containing the $\rho(\Gamma)$-fixed point $\left[\left(L^{*}\right)^{\perp}\right]$. Select a coordinate system on $U \approx \mathbb{R}^{2}$ such that the intersection with $U$ of projective lines in $L^{*}$ are vertical lines $\{*\} \times \mathbb{R}$. Then, according to Corollary 3.12, $L \cap U$ is the graph of a continuous map from $\mathbb{R}$ into $\mathbb{R}$. Then, the lemma becomes obvious.

We are left with the case where $L, L^{*}$ are not projective lines, ie, the strongly irreducible case (cf Proposition 4.7). We will show that $P(V) \backslash L$ is a topological disc, which will be enough for the proof of the Lemma due to the symmetry of the problem.

Let $S(V)$ be the $N$-sphere of $V$ : the radial projection $\Pi_{S}: S(V) \rightarrow P(V)$ is the double covering, with covering automorphisms $\pm i d$. The group $\operatorname{SL}(V)$ acts naturally on $S(V)$ : consider the sphere $S(V)$ as the space of half-lines in $V$.

The limit curve $L$ is a simple closed curve. There are two cases:

- $\Pi_{S}^{-1}(L)$ is a connected simple closed curve $\hat{L}$, or

- $\Pi_{S}^{-1}(L)$ is the union of two connected simple closed curves $\hat{L}_{+}, \hat{L}_{-}$. 
In the first case, the lemma follows from Jordan's Theorem, and the (-id)-invariance of $\hat{L}$. Hence, the proof of the lemma amounts to excluding the second case, more precisely, to proving that in the second case, $\rho$ is hyperconvex.

The antipody - id exchanges $\widehat{L}_{+}$and $\widehat{L}_{-}$. For any element $\gamma$ of $\Gamma$, there is one and only one way to lift $\rho(\gamma)$ to an element $\hat{\rho}(\gamma)$ of $\operatorname{SL}(V)$ preserving $\widehat{L}_{+}$. It provides a representation $\hat{\rho}: \Gamma \rightarrow \operatorname{SL}(V)$.

For any $\left[u^{*}\right]$ in $L^{*}$, according to Corollary 3.12, the kernel of $u^{*} \in V^{*}$ intersects $\widehat{L}_{+} \cup \widehat{L}_{-}$in two points, one opposite the other. Since $\widehat{L}_{-}=-\widehat{L}_{+}$, the kernel of $u^{*}$ intersects $\hat{L}_{+}$in one and only one point $u\left(\left[u^{*}\right]\right)$ of $\hat{L}_{+}$: hence, the sign of $u^{*}$ on $\widehat{L}_{+} \backslash\left\{u\left(\left[u^{*}\right]\right)\right\}$ is constant. Select $u^{*} \in S\left(V^{*}\right)$ so that this sign is positive.

This process defines a way to simultaneously lift $L$ and $L^{*}$ in $V, V^{*}$ to closed subsets $\hat{L}_{+}, \hat{L}_{+}^{*}$ such that for any $\left(u, v^{*}\right)$ in $\hat{L}_{+} \times \widehat{L}_{+}^{*}$ we have $\left\langle u \mid v^{*}\right\rangle \geq 0$. In the terminology of [4], this means that $\Lambda$ is a positive subset of $X$. According to Proposition 1.2 of [4], since $\rho$ is strongly irreducible, $\rho(\Gamma)$ preserves a strictly convex domain of $P(V)$, meaning precisely that $\rho$ is hyperconvex, a contradiction.

An equivalent way to formulate Lemma 6.5 is:

Corollary 6.6 $A(G, Y)$-Anosov representation is hyperconvex if and only if the limit curve $L$ or $L^{*}$ is null-homotopic.

Corollary 6.7 Every nonhyperconvex Anosov flag representation can be continuously deformed to the trivial representation.

Proof According to [12], a nonhyperconvex Anosov representation does not belong to the Hitchin component. On the other hand, it lifts to a representation $\hat{\rho}: \Gamma \rightarrow P^{+} \mathrm{GL}(V)$. Indeed, keeping the notation used in the proof of Lemma 6.5, we can select a connected component $U^{+}$of $S(V) \backslash \widehat{L}$. We then define $\hat{\rho}(\gamma)$ as the unique lift in $P^{+} \mathrm{GL}(V)$ of $\rho(\gamma)$ preserving $U^{+}$. It provides the required representation $\hat{\rho}$. The corollary then follows from Section 2.6.

Corollary 6.8 For every $[u]$ in $P(V)$ there is an element $\left[v^{*}\right]$ in $L^{*}$ "containing" $[u]$, ie, such that $\left\langle u \mid v^{*}\right\rangle=0$.

Proof If not, there is an element $u_{0}$ of $V \backslash\{0\}$ such that $L^{*}$ is disjoint from $\left[u_{0}^{\perp}\right]$. The projective line $\left[u_{0}^{\perp}\right]$ then lifts to a great circle in $S\left(V^{*}\right)$ avoiding $\widehat{L}^{*}$. Hence, $\widehat{L}^{*}$ is contained in a hemisphere $U$. We obtain a contradiction since $\widehat{L}^{*}=-\widehat{L}^{*}$ and $U \cap(-U)=\varnothing$. 
Compare the following proposition with Remark 3.17 (for the notation, recall Definitions 3.15 and 3.16):

Proposition 6.9 $T$ and $T^{*}$ are topological Klein bottles, and $A, A^{*}$ are Möbius bands. The domain $\Omega$ is connected, homeomorphic to the solid torus $\Omega_{0} \approx \mathbb{S}^{1} \times \mathbb{R}^{2}$.

Proof By the Jordan-Schönflies Theorem, there is a homeomorphism $f$ of $P(V)$ mapping the simple closed curve $L$ on the projective line $L_{0}$. The circle bundle $\Pi: X \rightarrow P(V)$ and pullback bundle $f^{*} \Pi$ have the same Euler class; hence, $f$ lifts to some homeomorphism $F$ of $X$ into itself, preserving the fibers of $\Pi$, and inducing $f$ on $P(V)$. Then, $F(T)$ is the Klein bottle $T_{0}$. The first part of the proposition follows. The complement $W_{0}$ of $T_{0}$ in $P(V)$ is a solid torus, and $F\left(T^{*}\right) \cap W_{0}$ is an annulus. For a better visualisation, lift to the 4 -sheeted covering $\hat{X} \subset S(V) \times S\left(V^{*}\right)$ : we are led to the well-known fact that the complement, in a compact solid torus with boundary $\widehat{W}_{0}$, of a compact annulus with boundary topologically embedded admitting as boundary two disjoint essential curves of $\partial \widehat{W}_{0}$, is the union of two solid tori.

Corollary 6.10 The flag manifold $M=\rho(\Gamma) \backslash \Omega$ is homeomorphic to a circle bundle over $\Sigma$.

Proof According to Proposition 6.9, if $\widehat{\Gamma}$ denote the fundamental group of $M$, we have an exact sequence

$$
0 \rightarrow \mathbb{Z} \rightarrow \widehat{\Gamma} \rightarrow \Gamma \rightarrow 0
$$

where $\mathbb{Z}$ is the fundamental group of the solid torus $\Omega$. Hence, $M$ is sufficiently large (its first homology group is infinite), is $\mathbb{R} P^{2}$-irreducible (its universal covering is homeomorphic to $\mathbb{R}^{3}$ ), and is homotopically equivalent to a circle bundle over $\Sigma$. The corollary follows from [19].

\subsection{Minimality of tautological foliations}

Recall that a 1-dimensional foliation is minimal if all the leaves are dense. In this section we prove Theorem 1.3:

Theorem 1.3 Let $\rho$ be a $(G, Y)$-Anosov representation, which is not hyperconvex.

- If $L$ (respectively $L^{*}$ ) is a projective line, then the second (respectively first) tautological foliation is topologically conjugate to the horocyclic flow of a Desarguian Anosov flow,

- If $L$ is not a projective line, then the second tautological foliation is not minimal. 
Sketch of proof If $L^{*}$ is a projective line, then the representation is hyperbolic: the Theorem reduces in this case to Proposition 6.4. By symmetry, it also applies to the case where $L$ is a projective line.

Assume now that $L$ is not a projective line. If $L^{*}$ is a projective line, Theorem $\mathrm{C}$ of [2] states precisely that the second tautological foliation is not minimal. Now we briefly review how the arguments of Section 5.2 of [2] still applies even if $L^{*}$ is not a projective line: consider the set of flags $\left([u],\left[u^{*}\right]\right) \in \Omega$ such that $\left[u^{*}\right] \cap L$ is reduced to a point. Its closure $\mathcal{M}$ is invariant by the second tautological flow. Arguments of Lemma 5.7 of [2] proves that $\mathcal{M}$ is not all of $\Omega$, and those of Lemma 5.8 of [2] show that if $\mathcal{M}$ were empty, then the second tautological foliation would be expansive: by [6], it would be topologically conjugate to a finite covering of some geodesic flow. This leads to a contradiction, as in [2].

\section{The invariant Möbius bands}

In this section, we consider a nonhyperconvex Anosov flag representation $\rho$. The action of $\rho(\Gamma)$ on $\Lambda$ is conjugate to the usual projective action on $\mathbb{R} P^{1}$. The action on $\Omega$ is proper, topologically conjugate to the action of $\Gamma$ on $H$ by left translations, at least when $\rho$ is a deformation of a canonical flag representation inside the space of Anosov flag representations (see Question 2 in Section 8).

But the dynamics on the invariant Möbius bands are much more subtle. When $L$ is a projective line $\left[\left(u_{0}^{*}\right)^{\perp}\right]$, it follows from Corollary 3.12 that $\left[u_{0}^{*}\right]$ does not belong to $L^{*}$. Hence, the flags $\left([u],\left[u_{0}^{*}\right]\right)$ with $[u] \in L$ form a continuous $\rho(\Gamma)$-invariant curve in $A$. Conversely:

Proposition 7.1 Consider the Fuchsian projective action of $\Gamma \subset H$ on the projective line $\mathbb{R} P^{1}$ and the $\Gamma$-action on $A$ induced by $\rho$. If there is a $\Gamma$-equivariant measurable map $\sigma: \mathbb{R} P^{1} \rightarrow A$, then the limit curve $L$ is a projective line.

Proof This is essentially the content of Lemma 4.17 of [2], that we reproduce here: let $\overline{\mathcal{Q}}$ be the complement of the diagonal in $\mathbb{R} P^{1} \times \mathbb{R} P^{1}$ (see Section 2.4.2). The diagonal action of $\Gamma$ on $\overline{\mathcal{Q}}$ is ergodic for some $\Gamma$-invariant measure equivalent to the Lebesgue measure and preserved by the flip map $(x, y) \mapsto(y, x)$ (for example, the projection on the orbit space of the geodesic flow of the Liouville measure). We say that a subset of $\overline{\mathcal{Q}}$ is conull if the Lebesgue measure of its complement is zero. The crucial and classical observation is that this ergodicity property implies that there is no measurable equivariant map from $\overline{\mathcal{Q}}$ into a topological space on which $\Gamma$ acts freely and properly discontinuously. 
Decompose $\sigma: \mathbb{R} P^{1} \rightarrow A$ into two functions $\eta: \mathbb{R} P^{1} \rightarrow L$ and $\eta^{*}: \mathbb{R} P^{1} \rightarrow P\left(V^{*}\right) \backslash L^{*}$.

Assume that the set of pairs $(x, y)$ for which $\eta(y)$ does not belong to $\eta^{*}(x)$ is conull. Then, its intersection with its image by the flip map is conull, and its intersection with all its $\Gamma$-iterates is too. Thus, there is a conull $\Gamma$-invariant subset $\mathcal{E}$ of $\overline{\mathcal{Q}}$ of pairs $(x, y)$ for which the projective lines $\eta^{*}(x)$ and $\eta^{*}(y)$ intersect at some point $[u(x, y)]$ different from $\eta(x)$ and $\eta(y)$. We have then two cases: either almost every $[u(x, y)]$ belongs to $L$, or almost all of them belong to $P(V) \backslash L$. In the first case, $(x, y) \mapsto\left(\eta_{+}^{-1}(\eta(x)), \eta_{+}^{-1}(\eta(y)), \eta_{+}^{-1}([u(x, y)])\right)$ is a $\Gamma$-equivariant map from $\mathcal{E}$ into the set of distinct triples of points of $\mathbb{R} P^{1}$. Since the action of $\Gamma$ on this set of triples is free and properly discontinuous, we obtain a contradiction with the ergodic argument presented above. In the second case, the map associating to a pair $(x, y)$ the flag $\left([u(x, y)], \eta^{*}(x)\right)$ is a $\Gamma$-equivariant map from $\mathcal{E}$ into $\Omega$. We obtain once more a contradiction with the ergodic argument by Theorem 1.2.

Therefore, the set of pairs $(x, y)$ for which the line $\eta^{*}(x)$ contains $\eta(y)$ is conull. For every $x$, denote by $E_{x}$ the set of those $y$ so that $\eta(y)$ belongs to $\eta^{*}(x)$. By Fubini's Theorem, there is a set $U$ of full measure in $\mathbb{R} P^{1}$ such that for every $x$ in $U, E_{x}$ has full measure. Since $\Gamma$ is countable, we can as well assume that $U$ is $\Gamma$-invariant. Let $z$ and $x$ be two distinct points in $U$. By construction, $\eta^{*}(x) \cap \eta^{*}(z)$ contains $\eta\left(E_{x} \cap E_{z}\right)$. Since $\eta$ is not almost everywhere constant the intersection $\eta^{*}(x) \cap \eta^{*}(z)$ is not reduced to a point. In other words, $\eta^{*}(x)=\eta^{*}(z)$ and therefore $\eta^{*}$ is constant on $U$. Therefore $\eta^{*}(U)$ is a projective line fixed by $\rho(\Gamma)$. Hence, $\rho$ is hyperbolic, and $L$ is the projective line $\eta^{*}(U)$.

Of course, the similar lemma with $A$ replaced by $A^{*}$ is true.

Corollary 7.2 If $\rho$ is strongly irreducible, the maps $\eta_{ \pm}$are not Lipschitz.

Proof Assume that $\eta_{+}$is Lipschitz. Then, it is differentiable almost everywhere. Its differential defines a measurable map $\sigma=\left(\eta, \eta^{*}\right): \mathbb{R} P^{1} \rightarrow X$, where the first component $\eta$ is $\eta_{+}$, and the second component $\eta^{*}$ is the projective line tangent to the image of the differential of $\eta_{+}$. According to Proposition 7.1, the second component $\eta^{*}(x)$ is an element of $L^{*}$ for almost every $x$. According to Corollary 3.12, we have $\eta^{*}(x)=\eta_{-}(x)$. More precisely, it follows from this Corollary 3.12 that $L$ is locally strictly convex. We obtain a contradiction since $\rho$ was assumed to be nonhyperconvex.

The same proof applies to $\eta_{-}$. 


\section{Open questions}

Let $\rho: \Gamma \rightarrow G$ be a nonhyperconvex Anosov flag representation. Here, we have essentially answered Question 8 of [2] by Theorem 1.2. We also answered Question 6: the curve $\Lambda$ is Hölder continuous. But some interesting questions are still open:

Question 1 Is the circle bundle $\rho(\Gamma) \backslash \Omega$ homeomorphic to $\Gamma \backslash H$, ie, to the double covering of the unit tangent bundle of $\Sigma=\Gamma \backslash \mathbb{H}^{2}$ ?

According to [12], the space of hyperconvex flag representations is connected: it is the entire Hitchin component. Thus, we can wonder:

Question 2 Is the space of nonhyperconvex Anosov flag representations connected?

If the answer to Question 2 is yes, then the answer to Question 1 is yes.

Question 3 Let $\rho^{\prime}: \Gamma \rightarrow G$ be another nonhyperconvex Anosov flag representation. Assume that the $\rho^{\prime}(\Gamma)$-action on the invariant Möbius band $A^{\prime}$ (respectively $\left(A^{\prime}\right)^{*}$ ) is topologically conjugate to the $\rho(\Gamma)$-action on $A$ (resp. $A^{*}$ ). Does that imply that $\rho^{\prime}$ and $\rho$ are conjugate in $G$ ?

Observe that according to Proposition 6.3, Proposition 6.4 and Proposition 7.1, the answer to this question is yes when $\rho$ is a hyperbolic radial representation!

Question 4 Are the tautological foliations associated to nonhyperconvex Anosov flag representations uniquely ergodic?

Concerning dynamical properties of tautological foliations, recall Questions 1 and 5 of [2]:

Question 5 Can a tautological foliation associated to a nonhyperconvex Anosov flag representation admit a periodic orbit? Can it have nonzero entropy?

\section{Appendix: The $(G, Y)$-Anosov property for linear deforma- tions}

In this Appendix, we prove that horocyclic representations and their $u$-deformations are $(G, Y)$-Anosov. We first consider horocyclic representations. The proof of Theorem 2.11 we produce here is quite sophisticated, but it is a necessary preparation for the most delicate case of $u$-deformations. 


\subsection{Horocyclic representations}

We define a map $f: H \rightarrow Y$ by $f(h)=\left(\left[\rho_{0}(h) e_{1}\right],\left[e_{2}\right],\left[\rho_{0}(h) e_{3}\right]\right)$. It is a $\rho_{0}(\Gamma)-$ equivariant map, defining a section $s$ of $\pi_{\rho}$. Our task is to prove that $s$ defines a $(G, Y)$-Anosov structure.

Consider $f_{+}=\pi_{+} \circ f$ : it provides a section $s^{+}$of $\pi_{\rho_{0}}^{X}$. It can be written $f_{+}(h)=$ $\left(\left[\rho_{0}(h) e_{1}\right],\left[\rho_{0}^{*}(h) e_{3}^{*}\right]\right)$.

We first consider the first component $\left[\rho_{0}(h) e_{1}\right]$, which defines a section $s_{P}^{+}$of the flat $P(V)$-bundle associated to $\rho_{0}$ : this bundle $\pi_{P}: E_{\rho_{0}}(P) \rightarrow M$ is defined in the same way as the bundles $E_{\rho_{0}}$ and $E_{\rho_{0}}(X)$; it also admits a horizontal flow $\Phi_{P}^{t}$ above $\Phi^{t}$. Define $\Psi(h,(\alpha, \beta))=\left(h,\left[\rho_{0}(h) e_{1}+\alpha \rho_{0}(h) e_{2}+\beta \rho_{0}(h) e_{3}\right]\right)$. It is a $\Gamma$-equivariant map, when $H \times \mathbb{R}^{2}$ is equipped with the $\Gamma$-action $\gamma(h,(\alpha, \beta))=(\gamma h,(\alpha, \beta))$, and $H \times P(V)$ is equipped with the action $\gamma(h,[u])=\left(\gamma h,\left[\rho_{0}(\gamma) u\right]\right)$. Hence, it induces a fibered map $\bar{\Psi}: M \times \mathbb{R}^{2} \rightarrow E_{\rho_{0}}(P)$. More precisely, it provides a trivialisation of an open neighborhood $W$ of the image of $s_{P}^{+}$.

Select any left invariant metric $m$ on $H$. Equip $\mathbb{R}^{2}$ with the Euclidean norm $d \alpha^{2}+d \beta^{2}$. The image by $\Psi$ of the product metric is a $\Gamma$-invariant metric: it provides a metric on the neighborhood $W$. Now, we simply observe that the flow $\Phi_{P}^{t}$ is expressed in the chart $\bar{\Psi}$ by the simple expression:

$$
\Phi_{P}^{t}(p,(\alpha, \beta))=\left(\Phi^{t}(p),\left(e^{t} \alpha, e^{2 t} \beta\right)\right)
$$

It immediately follows that the image of $s_{P}^{+}$is a (exponentially) repellor of $\Phi_{P}^{t}$.

Similar reasoning on the second component $\left[\rho_{0}^{*}(h) e_{3}^{*}\right]$ of $f_{+}(h)$ shows that it provides a section of the flat $P\left(V^{*}\right)$-bundle associated to $\rho_{0}^{*}$ which is also a (exponentially) repellor of the corresponding horizontal flow.

Combining these two facts, we obtain that the image of $s_{P}^{+}$is an (exponentially) attractor for $\Phi_{X}^{t}$.

A completely similar argument establishes that the image of the section $s^{-}$of $E_{\rho_{0}}(X)$ furnished by $\pi_{-} \circ f$ is an (exponentially) attractor for $\Phi_{X}^{t}$. Of course, the pairs $\left(s^{+}(p), s^{-}(p)\right)$ all belong to $\mathcal{Y}_{\rho_{0}}$. It follows that $s \approx\left(s^{+}, s^{-}\right)$satisfies all the criteria required in Definition 2.10: it defines a $(G, Y)$-Anosov structure. Theorem 2.11 is proved.

\subsection{Linear $u$-deformations}

For a fixed inclusion $1: \Gamma \subset H$ and embedding $\rho_{0}: H \rightarrow G$, any $u \in H^{1}(\Gamma, \mathbb{R})$ defines a representation $\rho_{u}$. We assume here that the stable norm $|u|_{s}$ is less than $1 / 2$, and we want to prove that $\rho_{u}$ is $(G, Y)-$ Anosov. 
Since $\varphi^{t}$ preserves the point $\left[e_{2}\right]$ and acts trivially on $L=\left[\left(e_{2}^{*}\right)^{\perp}\right]$, the map $f(h)=$ $\left(\left[\rho_{0}(h) e_{1}\right],\left[e_{2}\right],\left[\rho_{0}(h) e_{3}\right]\right)$ is still $\rho_{u}(\Gamma)$-equivariant: it provides the suitable section $s$.

Exactly as we did for horocyclic representations, we study separately the two components $E_{\rho_{u}}(X)$. Each of them decomposes as a sum of subbundles $E_{\rho_{u}}(P), E_{\rho_{u}}\left(P^{*}\right)$. At the end, we have to consider 4 sections of bundles over $M$ by projective spaces, and we have to prove that the images of these sections are repellors or attractors of the associated horizontal flows.

We only discuss here the section of $E_{\rho_{u}}(P)$ defined by $h \mapsto\left[\rho_{0}\left(e_{1}\right)\right]$. The other sections can be treated in a similar way left to the reader.

We consider the map $\Psi(h,(\alpha, \beta))=\left(h,\left[\rho_{0}(h) e_{1}+\alpha \rho_{0}(h) e_{2}+\beta \rho_{0}(h) e_{3}\right]\right)$. The main difference with the horocyclic case is that the $\Gamma$-action on $H \times \mathbb{R}^{2}$ to be considered is:

$$
\gamma(h,(\alpha, \beta))=\left(\gamma h,\left(e^{-u(\gamma)} \alpha, \beta\right)\right)
$$

The quotient of this action is then an $\mathbb{R}^{2}$-bundle $E$ over $M$, and we have a fibered map $\bar{\Psi}: E \rightarrow E_{\rho_{u}}(P)$. The image of $\bar{\Psi}$ is a neighborhood of the $\Phi_{P}^{t}$-invariant section to be studied.

The key point is to define a metric on $H \times \mathbb{R}^{2}$ such that:

- the $\Gamma$-action defined by Equation (3) is isometric,

- the null section $h \mapsto(h, 0)$ is a repellor for the horizontal flow $(h,(\alpha, \beta)) \mapsto$ $\left(h a^{t},\left(e^{t} \alpha, e^{2 t} \beta\right)\right)$.

Let $\Sigma$ be the Riemannian surface $\Gamma \backslash \mathbb{H}^{2}$. The cohomology class $u \in H^{1}(\Gamma, \mathbb{R})$ can be represented by a 1 -form $\omega$ on $\Sigma$ such that the integration of $\omega$ along any loop representing an element $\gamma$ of $\Gamma \approx \pi_{1}(\Sigma)$ is $u(\gamma)$.

The quotient $\Gamma \backslash \bar{H}$ is naturally identified with the unit tangent bundle of $\Sigma$ : the orbit space of the left action of $\mathrm{SO}(2)$ on $\bar{H}$ is canonically identified with $\Sigma$. Denote by $\eta: \Gamma \backslash \bar{H} \rightarrow \Gamma \backslash \bar{H} / \mathrm{SO}(2)$ the quotient map, and consider the 1 -form $\eta^{*}(\omega)$. The assumption $|u|_{s}<1 / 2$ implies the following ${ }^{4}$ : the absolute value of the integration of $\eta^{*}(\omega)$ along a periodic orbit of $\bar{\Phi}^{t}$ with period $T$ is less than $C T$, for $C=2|u|_{s}<1$. Any orbit of $\Phi^{t}$ can be approximated by periodic orbits. Therefore, if $\bar{\theta}:[0, T] \rightarrow \bar{M}$ is a portion of an orbit of $\bar{\Phi}^{t}$ (ie, $\bar{\theta}(t)=\bar{\Phi}^{t}(p)$ for some $p$ ), we have:

$$
\left|\int_{\bar{\theta}} \eta^{*}(\omega)\right| \leq C T
$$

\footnotetext{
${ }^{4}$ Recall that $\bar{\Phi}^{t}$ is the geodesic flow with parametrisation multiplied by 2 (see Remark 2.5): this factor 2 compensates for the stable norm $1 / 2$.
} 
Let $\widehat{\omega}$ be the lifting of $\eta^{*}(\omega)$ to $M=\Gamma \backslash H$. If $\theta(t)=\Phi^{t}(p)$, we have:

$$
\left|\int_{\theta} \hat{\omega}\right| \leq C T
$$

Finally, the lifting $\tilde{\omega}$ in $H$ of $\widehat{\omega}$ is exact: there is a function $v: H \rightarrow \mathbb{R}$ such that $d v=\widetilde{\omega}$. According to Equation (4), we have:

$$
\left|v\left(h a^{t}\right)-v(h)\right| \leq C t
$$

Equip the fiber $\mathbb{R}^{2}$ over $h \in H$ with the metric $e^{2 v(h)} d \alpha^{2}+d \beta^{2}$ : it gives a metric on $H \times \mathbb{R}^{2}$. Since

$$
v(\gamma h)-v(h)=\int_{[h, \gamma h]} d v=\int_{[h, \gamma h]} \tilde{\omega}=u(\gamma),
$$

the transformations defined in Equation (3) are isometries. Now, it follows from Equation (5) that the horizontal flow $(h,(\alpha, \beta)) \mapsto\left(h a^{t},\left(e^{t} \alpha, e^{2 t} \beta\right)\right)$ expands the norm on the fibers by a factor at least $e^{(1-C) t}$. Theorem 4.2 follows.

\section{References}

[1] V Bangert, Minimal geodesics, Ergodic Theory Dynam. Systems 10 (1990) 263-286 MR1062758

[2] T Barbot, Flag structures on Seifert manifolds, Geom. Topol. 5 (2001) 227-266 MR1825662

[3] T Barbot, Plane affine geometry and Anosov flows, Ann. Sci. École Norm. Sup. (4) 34 (2001) 871-889 MR1872423

[4] Y Benoist, Automorphismes des cônes convexes, Invent. Math. 141 (2000) 149-193 MR1767272

[5] R Bowen, B Marcus, Unique ergodicity for horocycle foliations, Israel J. Math. 26 (1977) 43-67 MR0451307

[6] M Brunella, Expansive flows on Seifert manifolds and on torus bundles, Bol. Soc. Brasil. Mat. (N.S.) 24 (1993) 89-104 MR1224301

[7] S Choi, W M Goldman, Convex real projective structures on closed surfaces are closed, Proc. Amer. Math. Soc. 118 (1993) 657-661 MR1145415

[8] A Fathi, F Laudenbach, V Poenaru (editors), Travaux de Thurston sur les surfaces, Astérisque 66-67, Soc. Math. France, Paris (1979) MR568308 Séminaire Orsay, With an English summary

[9] É Ghys, Flots d'Anosov dont les feuilletages stables sont différentiables, Ann. Sci. École Norm. Sup. (4) 20 (1987) 251-270 MR911758 
[10] V L Ginzburg, B Z Gürel, $A C^{2}$-smooth counterexample to the Hamiltonian Seifert conjecture in $\mathbb{R}^{4}$, Ann. of Math. (2) 158 (2003) 953-976 MR2031857

[11] W M Goldman, Geometric structures on manifolds and varieties of representations, from: "Geometry of group representations (Boulder, CO, 1987)", (W M Goldman, A R Magid, editors), Contemp. Math. 74, Amer. Math. Soc. (1988) 169-198 MR957518

[12] O Guichard, Composantes de Hitchin et représentations hyperconvexes de groupes de surface, J. Differential Geom. 80 (2008) 391-431 MR2472478

[13] G Kuperberg, A volume-preserving counterexample to the Seifert conjecture, Comment. Math. Helv. 71 (1996) 70-97 MR1371679

[14] K Kuperberg, A smooth counterexample to the Seifert conjecture, Ann. of Math. (2) 140 (1994) 723-732 MR1307902

[15] F Labourie, Anosov flows, surface groups and curves in projective space, Invent. Math. 165 (2006) 51-114 MR2221137

[16] F Salein, Variétés anti-de Sitter de dimension 3 possédant un champ de Killing non trivial, C. R. Acad. Sci. Paris Sér. I Math. 324 (1997) 525-530 MR1443988

[17] R Schwartz, Pappus' theorem and the modular group, Inst. Hautes Études Sci. Publ. Math. (1993) 187-206 MR1259431

[18] W P Thurston, Three-dimensional geometry and topology. Vol. 1, Princeton Math. Ser. 35, Princeton Univ. Press (1997) MR1435975 Edited by S Levy

[19] F Waldhausen, On irreducible 3-manifolds which are sufficiently large, Ann. of Math. (2) 87 (1968) 56-88 MR0224099

LANLG, EA 2151, Université d'Avignon

33, rue Louis Pasteur, F-84000 Avignon, France

thierry.barbot@univ-avignon.fr

Proposed: Martin Bridson

Seconded: Danny Calegari, David Gabai

Received: 25 April 2007

Revised: 14 October 2008 